\title{
An ALMA study of outflow parameters of protoclusters: outflow feedback to maintain the turbulence
}

\author{
T. Baug, ${ }^{1,2,3 \star}$ Ke Wang,,${ }^{1,3}+$ Tie Liu, ${ }^{4}$ Yue-Fang Wu, ${ }^{5}$ Di Li, ${ }^{6,7,8}$ Qizhou Zhang, ${ }^{9}$ Mengyao Tang, ${ }^{10}$ \\ Paul F. Goldsmith, ${ }^{11}$ Hong-Li Liu, ${ }^{12}$ Anandmayee Tej,${ }^{13}$ Leonardo Bronfman, ${ }^{14}$ L. Viktor Toth,${ }^{15}$ \\ Kee-Tae Kim, ${ }^{16}$ Shang-Huo Li, ${ }^{4}$ Chang Won Lee, ${ }^{16,17}$ Ken'ichi Tatematsu, ${ }^{18}$ and Tomoya Hirota ${ }^{18}$ \\ ${ }^{1}$ Kavli Institute for Astronomy and Astrophysics, Peking University, 5 Yiheyuan Road, Haidian District, Beijing 100871, China \\ ${ }^{2}$ S. N. Bose National Centre for Basic Sciences, Block-JD, Sector-III, Salt Lake, Kolkata 700106, India \\ ${ }^{3}$ PKU - SHAO Joint Research Center for Astrophysics, Peking University, 5 Yiheyuan Road, Haidian District, Beijing 100871, China \\ ${ }^{4}$ Shanghai Astronomical Observatory, Chinese Academy of Sciences, 80 Nandan Road, Shanghai 200030, China \\ ${ }^{5}$ Department of Astronomy, Peking University, 100871, Beijing, People's Republic of China \\ ${ }^{6}$ CAS Key Laboratory of FAST, National Astronomical Observatories, Chinese Academy of Sciences, Beijing 100101, China \\ ${ }^{7}$ University of Chinese Academy of Sciences, Beijing 100049, China \\ ${ }^{8}$ NAOC-UKZN Computational Astrophysics Centre, University of KwaZulu-Natal, Durban 4000, South Africa \\ ${ }^{9}$ Harvard-Smithsonian Center for Astrophysics, 60 Garden Street, Cambridge, MA 02138, USA \\ ${ }^{10}$ Department of Astronomy, Yunnan University, Kunming, 650091, China \\ ${ }^{11}$ Jet Propulsion Laboratory, National Aeronautics and Space Administration, United States \\ ${ }^{12}$ Departamento de Astronomía, Universidad de Concepción, Av. Esteban Iturra s/n, Distrito Universitario, 160-C, Chile \\ ${ }^{13}$ Indian Institute of Space Science and Technology, Thiruvananthapuram 695 547, Kerala, India \\ ${ }^{14}$ Departamento de Astronomía, Universidad de Chile, Casilla 36-D, Santiago, Chile \\ ${ }^{15}$ Eövös Loránd University, Department of Astronomy, Pázmány Péter sétány 1/A, H-1117, Budapest, Hungary \\ ${ }^{16}$ Korea Astronomy and Space Science Institute, 776 Daedeokdae-ro, Yuseong-gu, Daejeon 34055, Republic of Korea \\ ${ }^{17}$ University of Science and Technology, Korea (UST), 217 Gajeong-ro, Yuseong-gu, Daejeon 34113, Republic of Korea \\ ${ }^{18}$ National Astronomical Observatory of Japan, National Institutes of Natural Sciences, 2-21-1 Osawa, Mitaka, Tokyo 181-8588, Japan
}

Accepted XXX. Received YYY; in original form ZZZ

\begin{abstract}
With the aim of understanding the role of outflows in star formation, we performed a statistical study of the physical parameters of outflows in eleven massive protoclusters associated with ultra-compact HII regions. A total of 106 outflow lobes are identified in these protoclusters using the ALMA CO (3-2), $\mathrm{HCN}(4-3)$ and $\mathrm{HCO}^{+}(4-3)$ line observations. Although the position angles of outflow lobes do not differ in these three tracers, $\mathrm{HCN}$ and $\mathrm{HCO}^{+}$tend to detect lower terminal velocity of the identified outflows compared to CO. The majority of the outflows in our targets are young with typical dynamical time-scales of $10^{2}-10^{4}$ years, and are mostly composed of low-mass outflows along with at least one high-mass outflow in each target. An anti-correlation of outflow rate with dynamical time-scale indicates that the outflow rate possibly decreases with time. Also, a rising trend of dynamical time-scale with the mass of the associated core hints that the massive cores might have longer accretion histories than the low mass cores. Estimation of different energies in these protoclusters shows that outflows studied here cannot account for the generation of the observed turbulence, but can sustain the turbulence at the current epoch as the energy injection rate from the outflows is similar to the estimated dissipation rate.
\end{abstract}

Key words: ISM: clouds - ISM: jets and outflows - stars: formation - radio lines: ISM

\section{INTRODUCTION}

Star formation is a complex process that involves the collapse and accretion of gas onto protostars (Lada 1985). Molecular outflows are ubiquitous in the early stages of star formation. It is expected that at the protostellar phase a fraction of the accreted material from the envelope/disk is expelled as a result of angular momentum conservation

\footnotetext{
^ E-mail: tapas.polo@gmail.com (TB)

$\dagger$ Corresponding Author: kwang.astro@pku.edu.cn (KW)
}

(see reviews by Frank et al. 2014; Bally 2016). The gas is typically ejected in the form of high-velocity collimated jets that sweep up the surrounding material and form molecular outflows around the jet axis (Lee et al. 2000; Arce et al. 2007; Hartmann et al. 2016, and references therein). Compared to accretion disks, these outflows are more easily detectable in star-forming clouds (Bally 2016, and references therein). Since the first-ever observational detection of a molecular outflow in Orion A (Kwan \& Scoville 1976; Zuckerman et al. 1976), many outflows have been found in Galactic star-forming regions. Over the past few decades, studies of the outflows have increased 
significantly (see the review by Bally 2016), and the jets/outflows were detected in all mass regimes, starting from brown dwarfs (e.g., Whelan et al. 2005; Riaz et al. 2017) to intermediate-mass protostars (e.g., Zapata et al. 2010; Reiter et al. 2017; Takahashi et al. 2019) to high-mass protostars (e.g., Caratti o Garatti et al. 2015; Li et al. 2019, and references therein).

One of the main debates in the star formation community is whether the massive young stellar objects are a scaled-up version of low-mass young stellar objects (YSOs) where disk-accretion plays the dominating role for gaining the stellar mass. For the formation of low-mass stars, bipolar outflows driven by the accretion disks are proposed to be the basic formation mechanism theoretically (Shu et al. 1987), and are also verified observationally (e.g., Bontemps et al. 1996; Richer et al. 2000; Arce et al. 2007, and references therein). However, on the other hand understanding of the formation mechanism of massive stars is still elusive (Tan et al. 2014). Two major competing models for massive star formation are (i) core accretion via disk (McKee \& Tan 2003) and (ii) competitive accretion (Bonnell et al. 2001). The most obvious way to distinguish between these two models might be the detection of the accretion disk around massive protostars. But a direct detection of accreting disk is difficult because the accretion disk is small and short-lived, and also because of complicated gas dynamics at that scale (Kim \& Kurtz 2006). Here, the study of the properties of molecular outflows which are the manifestation of disk-accretion in young sources, could help us to improve our understanding of the underlying formation process (Shepherd \& Churchwell 1996; Beuther et al. 2002; Molinari et al. 2002; Arce et al. 2007). If it is assumed that massive stars do form via an accretion disk similar to the low-mass stars, they should generate massive and powerful outflows (see de Villiers et al. 2014, and references therein). A few recent studies (e.g., de Villiers et al. 2014; Li et al. 2018) indeed found the applicability of the same scaling between outflow activity and clump masses for both low-mass and massive objects, suggesting a similar formation mechanism. However, an extensive study of molecular outflows toward massive star-forming regions is still lacking owing to their large distances and high level of clustering. Recent interferometric observations with the ALMA enable us to target such regions thanks to its high spatial resolution and sensitivity. Studies of outflows associated with Galactic massive star-forming regions may provide us clues to better understand the launching mechanism of molecular outflows, and hence, the underlying star formation mechanism.

The outflows also inject a large amount of mechanical energy into the parent molecular cloud (Solomon et al. 1981; Lada 1985; Bachiller 1996). Such energetic feedback from young stars may significantly influence the self-regulation of star formation (Franco 1983). Feedback may provide the required turbulence to the parent molecular cloud for stabilizing it against the gravitational collapse (Shu et al. 1987; Nakamura \& Li 2007; Carroll et al. 2009; Matzner \& Jumper 2015). The impact of outflows on surrounding gas has been studied in several massive and low-mass star-forming regions (Arce et al. 2010; Nakamura et al. 2011; Narayanan et al. 2012; Li et al. 2015; Feddersen et al. 2020, and references therein). Majority of these studies reported that the outflows do not have sufficient energy to sustain the observed turbulence in their parent molecular clouds (see e.g., Li et al. 2020, and references therein). In a survey on Perseus molecular cloud, Arce et al. (2010) found that even though outflows have a large impact on the local clouds near the active star-forming area, the energy from outflows is not sufficient to produce the observed turbulence in the entire Perseus complex. A similar result was also found by Narayanan et al. (2012) and Li et al. (2015) for Taurus region. However, Nakamura et al. (2011) found that the protostellar outflows play a crucial role in replenishing the supersonic turbulence in $\rho$ Ophiuchi Main Cloud. Plunkett et al. (2013) also found the outflow energy comparable to the turbulent energy in NGC 1333 star-forming region. A similar result was also obtained by Yang et al. (2018) in hundreds of star-forming clumps.

Protostellar outflows have been extensively studied using several different atomic and molecular tracers at different wavelength regime, for example, $\mathrm{H}_{2}$, [FerI], $\mathrm{CO}, \mathrm{SiO}, \mathrm{HCN}, \mathrm{HCO}+, \mathrm{HNCO}, \mathrm{CS}$, and so on (see Bally 2016, for a detailed discussion). The emission lines of $\mathrm{CO}$, particularly the low- $J$ pure rotational transitions $(J \leq 4)$ in millimeter and sub-millimeter bands are widely used as tracers of outflow activity (Shepherd \& Churchwell 1996; Beuther et al. 2002; Yang et al. 2018). This is because of the high abundance of CO and also because observations of these $\mathrm{CO}$ lines are relatively straightforward using ground-based telescopes. In this paper, we report a study of outflow parameters derived from the $\mathrm{CO}(J=3-2)$ line toward 11 Galactic massive protoclusters $\left(1-24 \times 10^{3} \mathrm{M}_{\odot}\right.$; Liu et al. 2016) using data from ALMA. The details of these 11 target regions have been presented in Table 1 of our previous paper (Baug et al. 2020, hereafter Paper I). In total, we identified 106 lobes in these 11 target regions. A study of the orientation of the outflow lobes with respect to their host filaments is already reported in Paper I. The present paper concentrates on the derived outflow parameters (e.g., outflow mass, outflow rate, mechanical luminosity, dynamical timescale of outflow, etc.), their relation with the mass of the host cores, and the energy injection by these outflows to their parent clouds.

The paper is organized in the following manner. In Section 2, we present a brief introduction to the data. Identification of outflow lobes, comparison of the outflow parameters obtained with different tracers (i.e., $\mathrm{CO}(3-2), \mathrm{HCN}(4-3), \mathrm{HCO}^{+}(4-3)$; hereafter $\mathrm{CO}, \mathrm{HCN}$ and $\mathrm{HCO}^{+}$throughout the paper), and derivation of physical parameters of the CO outflows are presented in Section 3. A comparison of the $\mathrm{CO}$ outflow parameters with the parameters of the host cores and analysis on the energy budget of the host clouds are presented in Section 4. A conclusion of this study is presented in Section 5.

\section{DATA}

\subsection{ALMA observations}

Details of the ALMA observations (project 2017.1.00545.S; PI: Tie Liu) have been presented in Paper I. These data were observed as a pilot project for the ALMA Three-millimeter Observations of Massive Star-forming regions (ATOMS) survey (Liu et al. 2020a,b).

\section{RESULTS}

\subsection{Identification of Outflows}

The procedure to identify outflows adopted in this work was already reported in Paper I. For clarity, we briefly mention the procedure here. First, we cropped the data for the whole spectral window into smaller cubes that only cover $\pm 200 \mathrm{~km} \mathrm{~s}^{-1}$ centering on the systemic velocity of each target. We carefully examined these small positionposition-velocity (PPV) data cubes in SAOImageDS9 $9^{1}$ looking for the redshifted and blueshifted lobes around continuum sources starting from the highest velocity channels, as these channels are expected to be least contaminated by emission from the central clouds. The outflow lobes in a couple of regions are substantially crowded. There

\footnotetext{
1 https://sites.google.com/cfa.harvard.edu/saoimageds9
} 
were a few confusing $\mathrm{CO}$ outflow lobes that overlap with each other. For those, we compared the outflow lobes in different tracers (i.e., $\mathrm{CO}, \mathrm{HCN}, \mathrm{HCO}^{+}$, and $\mathrm{SiO}$ ) to confirm their credibility. However, in this paper we do not include any analysis from $\mathrm{SiO}$ observations. Analysis using this particular line will be reported for the full ATOMS survey sample in a future paper. Here, we considered as separate outflow lobes only those for which the emission between the two lobes at terminal velocity is separated by more than 5 pixels in the PPV space.

The terminal velocity and extent of each outflow lobe were considered up to a $5 \sigma$ level where $\sigma$ is the rms measured from a few line-free channels. In addition to the bipolar outflows, we identified unipolar outflows that are associated with continuum sources, and also a few outflow lobes without having any association with detected continuum sources. Here, we report a total of 106 outflow lobes. Among them 32 are bipolar and 42 are unipolar. Note that in this paper, we report one additional lobe which was confused and thought to be associated with another lobe in Paper I.

A total of 99 and 80 outflow lobes are detected in $\mathrm{HCN}$ and $\mathrm{HCO}^{+}$, respectively. Among the 106 lobes identified in $\mathrm{CO}, 80$ are identified in $\mathrm{HCN}$ and 62 are identified in $\mathrm{HCO}^{+}$. We also named the outflow lobes for convenience (see Tables 1 and 2). The naming of an outflow is done as follows. It contains the name of the region, followed by an alphanumeric character (that starts with the letter ' $\mathrm{O}$ ') to mark the source number, and a letter if multiple outflow lobes are identified with a single core. Details of all the identified outflow lobes, such as coordinates of the continuum source, orientations of outflow lobes on the plane of the sky, terminal velocity, and extent of each outflow are presented in Paper I and are thus not presented here. The outflow lobes identified with HCN overlaid on the $0.9 \mathrm{~mm}$ ALMA continuum maps for one region are presented in Figure 1. Figures for the identified outflows (in both $\mathrm{HCN}$ and $\mathrm{HCO}^{+}$) in the remaining regions are presented in Appendix A. The outflow lobes identified in $\mathrm{CO}$ are also marked by arrows in Figure 1. For reference, similar figures corresponding to the $\mathrm{CO}$ outflows are available in Paper I.

\subsection{Comparison of outflow lobes}

In order to examine how the detected outflow lobes conform in three different tracers, we plot the position angles, velocities, and extents of the outflow lobes in Figures 2 and 3.

As seen in Figure 2, the position angles of outflow lobes do not differ much in all three tracers and have typical variations $< \pm 10^{\circ}$. This particular variation is of the order of uncertainties in the measurements of the position angles. Figures $3 \mathrm{a}$ and $3 \mathrm{~b}$ show the projected extent of the outflow lobes in $\mathrm{HCN}$ and $\mathrm{HCO}^{+}$against $\mathrm{CO}$ emission, and the extent of the lobes against the terminal velocities of the outflow lobes in different tracers, respectively. Even though the extents of the lobes are generally found to be similar in $\mathrm{CO}$ and $\mathrm{HCN}$ (Figure 3a), a few $\mathrm{HCO}^{+}$lobes are found to be $10-20 \%$ shorter compared to the other two tracers. Also, $\mathrm{HCN}$ and $\mathrm{HCO}^{+}$typically have lower terminal velocities compared to those traced in CO. A similar result was noted earlier by López-Sepulcre et al. (2010). These authors found lower values of outflow momenta and kinetic energies estimated using $\mathrm{HCO}^{+}(1-0)$ emission compared to the commonly used tracers like $\mathrm{CO}$. Typically, $\mathrm{HCN}$ and $\mathrm{HCO}^{+}$trace the outflow in the vicinity of the driving source, while $\mathrm{CO}$ is sensitive enough to detect the low density and high-velocity outflow material. This is possibly because $\mathrm{CO}$ is more abundant than $\mathrm{HCN}$ and $\mathrm{HCO}^{+}$, and also because the excitation of $\mathrm{HCN}(4-3)$ and $\mathrm{HCO}^{+}(4-3)$ requires relatively higher temperatures compared to $\mathrm{CO}$ (3-2). Note that different tracers trace various components of the outflows, and

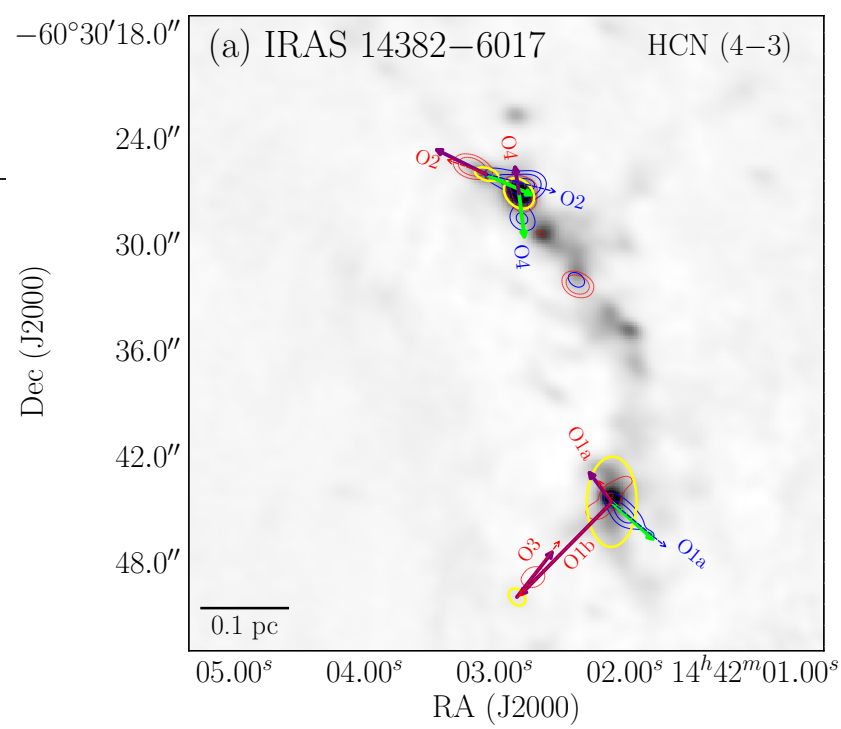

Figure 1. HCN outflow lobes toward the IRAS 14382-6017 region overlaid on the ALMA $0.9 \mathrm{~mm}$ continuum map of the region. The red and blue contours correspond to redshifted and blueshifted HCN emission integrated over carefully selected velocity ranges to depict the outflow lobes. The extents of blueshifted and redshifted outflow lobes in HCN are also marked by solid green and purple arrows, while the extent of outflow lobes identified using $\mathrm{CO}$ are shown by blue and red dashed arrows, respectively. The driving sources detected in the continuum map are marked in yellow ellipses.

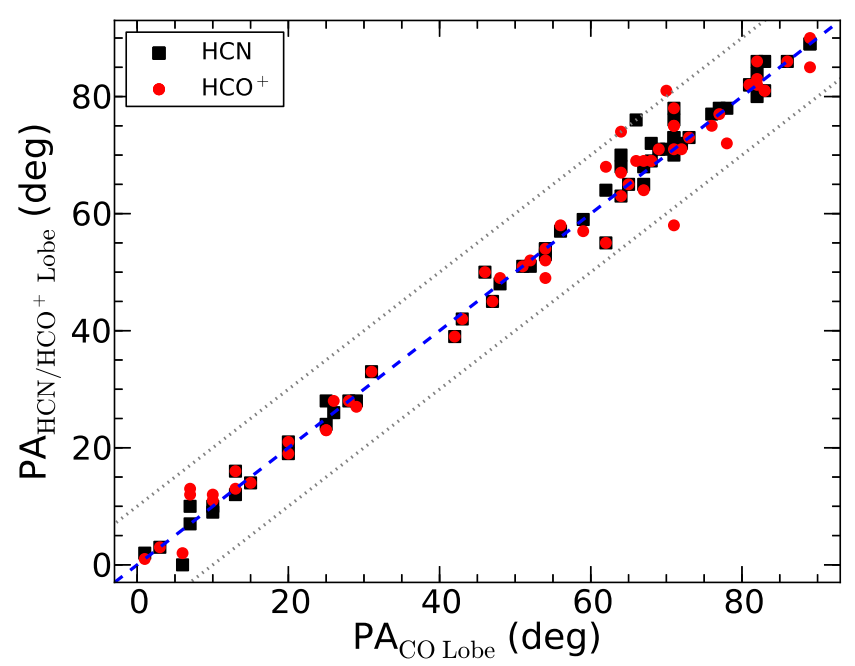

Figure 2. Comparison of the position angles of the lobes in HCN (black squares) and $\mathrm{HCO}^{+}$(red circles) with respect to the position angles of the lobes in $\mathrm{CO}$. The blue dashed line shows the ratio $\mathrm{PA}_{\mathrm{HCN} / \mathrm{HCO}^{+}} / \mathrm{PA}_{\mathrm{CO}}=1$. Grey dotted lines show the track for PA variation of $\pm 10^{\circ}$.

that explains the variation seen in the observed outflow parameters in three different tracers (see Bally 2016, for a detailed discussion).

\subsection{Dynamical properties of outflows}

In this paper, we only calculated the parameters for the outflows that are identified in $\mathrm{CO}$. The calculation of outflows parameters using $\mathrm{HCN}$ and $\mathrm{HCO}^{+}$are not performed in this paper. The physical properties of all the identified $\mathrm{CO}$ outflow lobes, such as mass 

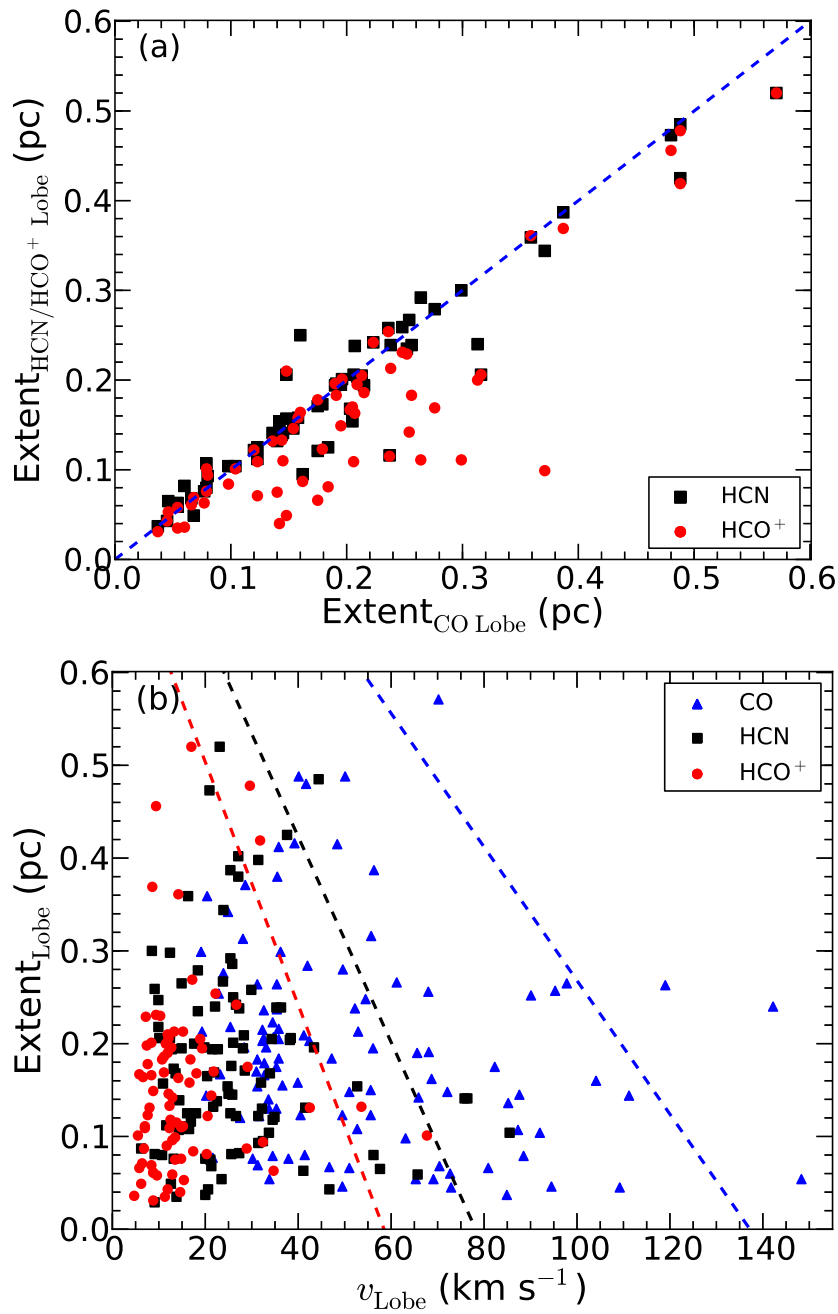

Figure 3. (a) Comparison between the extent of the outflow lobes in $\mathrm{HCN}$ and $\mathrm{HCO}^{+}$with respect to the extent of the outflow lobes in CO. (b) Comparison of the extent of the outflow lobes with the terminal velocity of the outflow. In both the panels, black square and red circles represent parameters for $\mathrm{HCN}$ and $\mathrm{HCO}^{+}$, respectively, while the blue triangles in the bottom panel represent the values for $\mathrm{CO}$. The blue, black and red dashed lines are drawn 'by-eye' for visualization (no fit involved) to portray typical limits and trends of terminal velocities and the extent of the lobes in $\mathrm{CO}, \mathrm{HCN}$ and $\mathrm{HCO}^{+}$, respectively.

$\left(M_{\text {out }}\right)$, momentum $\left(P_{\text {out }}\right)$, energy $\left(E_{\text {out }}\right)$, dynamical ages, outflow rate $\left(\dot{M}_{\text {out }}\right)$, mechanical luminosity $\left(L_{\text {mech }}\right)$, and outflow force $\left(F_{\text {out }}\right)$ were derived following the similar procedure described in Wang et al. (2011). We first simplified the equation given in Mangum \& Shirley (2015) to calculate the total CO column density of the outflows assuming that they are in local thermodynamic equilibrium and the emission is optically thin. Note that the calculated mass considering the emission to be optically thin might be underestimated by a factor of a few (see Offner et al. 2011; Arce et al. 2013; Dunham et al. 2014). However, opacity correction for the estimated mass of the outflows is primarily crucial if the lower bound velocity of the lobes are below $3 \mathrm{~km} \mathrm{~s}^{-1}$ (see Dunham et al. 2014, for a detailed discussion). We do not apply any opacity correction as the lower bound velocity of our idenntifed lobes are typically $\sim 5 \mathrm{~km} \mathrm{~s}^{-1}$. The equation for the total CO column density is given by :

$N_{\mathrm{CO}}^{\mathrm{thin}}\left(\mathrm{cm}^{-2}\right)=4.81 \times 10^{12}\left(T_{\mathrm{ex}}+0.92\right) \exp \left(\frac{33.12}{T_{\mathrm{ex}}}\right) \int T_{\mathrm{B}} d v$, where $T_{\mathrm{ex}}$ and $T_{\mathrm{B}}$ are excitation temperature and brightness temperature in $\mathrm{K}$, respectively, and $d v$ is in $\mathrm{km} \mathrm{s}^{-1}$. Estimated outflow parameters may vary depending on the adopted $T_{\mathrm{ex}}$. Dunham et al. (2014) performed a detailed calculation to estimate the correction factor for a $T_{\mathrm{ex}}$ range from $10-200 \mathrm{~K}$. They pointed out that low- $J$ transitions of $\mathrm{CO}$ (as in this study) are generally insensitive for $T_{\mathrm{ex}} \gtrsim$ $50 \mathrm{~K}$. Note that the targets in this study are massive star-forming regions associated with HII regions. Thus, a higher gas temperature is expected in these regions compared to the gas temperature typically assumed for cold clumps (10-30 K). Hence, we derived the outflow parameters in our target regions assuming a $T_{\mathrm{ex}}$ of $50 \mathrm{~K}$. Also, this is a crude assumption to consider a single $T_{\mathrm{ex}}$ for all the outflows. In fact, excitation temperatures may even vary over different parts of the lobes, and there may be very warm molecular gas in shocks (see e.g., Green et al. 2011, and references therein). Thus, consideration of a single $T_{\mathrm{ex}}$ can lead to significant underestimates (by up to factors of 3-4) in the outflow kinetic energy and mechanical luminosity which are the most sensitive to the highest-velocity gas (see Downes \& Cabrit 2007; Dunham et al. 2014, for a detail discussion). As discussed by Dunham et al. (2014), a different value of $T_{\mathrm{ex}}$ from $10-50 \mathrm{~K}$ might lead to a maximum variation in the outflow parameter by a factor of 1-3 which is of the order of uncertainty of these derived parameters. Here, we derived the outflow parameters using the equations given in Wang et al. (2011):

$$
\begin{aligned}
M_{\text {out }} & =d^{2}\left[\frac{\mathrm{H}_{2}}{\mathrm{CO}}\right] \bar{m}_{\mathrm{H}_{2}} \int_{\Omega} N_{\mathrm{CO}}^{\text {thin }}\left(\Omega^{\prime}\right) d \Omega^{\prime}, \\
P_{\text {out }} & =M_{\text {out }} v, \\
E_{\text {out }} & =\frac{1}{2} M_{\text {out }} v^{2}, \\
t_{\text {dyn }} & =\frac{L_{\text {flow }}}{v_{\text {Lobe }}} \\
\dot{M}_{\text {out }} & =\frac{M_{\text {out }}}{t_{\text {dyn }}} \\
L_{\text {mech }} & =\frac{E_{\text {out }}}{t_{\text {dyn }}}, \\
F_{\text {out }} & =\frac{P_{\text {out }}}{t_{\text {dyn }}},
\end{aligned}
$$

where $d$ is the distance to the source. $\left[\frac{\mathrm{H}_{2}}{\mathrm{CO}}\right]$ is the abundance ratio of $\mathrm{H}_{2}$ to $\mathrm{CO}$ which is taken to be $10^{4}$ (Blake et al. 1987). The mean molecular weight, $\bar{m}_{\mathrm{H}_{2}}$, is assumed to be $2.33 m_{\mathrm{H}}$. The parameter $\Omega$ is the solid angle subtended by the outflow lobe, and $v$ is the velocity of the outflow relative to the systemic velocity. The parameters $L_{\text {flow }}$ and $v_{\text {Lobe }}$ are the extent of the outflow lobe from the driving source and the outflow terminal velocity, respectively.

Since we only measure the radial component of the outflow velocity and the projected size of the lobe on the plane of the sky, it is important to include the corrections for the inclination. We have corrected the parameters, $L_{\text {flow }}, P_{\text {out }}, E_{\text {out }}, t_{\text {dyn }}, \dot{M}_{\text {out }}, L_{\text {mech }}$, and $F_{\text {out }}$ multiplying by $1 / \sin i, 1 / \cos i, 1 / \cos ^{2} i, \cos i / \sin i, \sin i / \cos i$, $\sin i / \cos ^{3} i$, and $\sin i / \cos ^{2} i$, respectively, for a mean inclination angle $(i)$ of 57.3 assuming all orientations are equally favorable (see Dunham et al. 2014, for a detailed description). However, the outflow parameters estimated here should be treated only as lower limits because it might include several observational biases. The assumption for the $\mathrm{CO}$ line to be optically thin might not always be true, and unlike other low-density gas tracers (e.g., $\mathrm{C}_{\mathrm{II}} 157 \mu \mathrm{m}$ line), $\mathrm{CO}$ is unable to trace the outflows in low-density regions. Also, a lower limit may be due to the limited sensitivity of our observations. Moreover, the lower bound velocity of our identified outflows is at about $5 \mathrm{~km} \mathrm{~s}^{-1}$ from the 
systemic velocity of the cloud because we could not separate out the outflow gas component from the host cloud below this velocity limit. However, this low velocity part of the outflow might have a significant contribution to the observed outflow mass (Arce \& Goodman 2001; Downes \& Cabrit 2007; Offner et al. 2011). Beside, it was not always possible to disentangle the overlapping emission from two nearby lobes, particularly when they approached the cloud velocity (within 2-6 $\mathrm{km} \mathrm{s}^{-1}$ ). Although, the minimum detectable velocity of our identified outflows is at about $5 \mathrm{~km} \mathrm{~s}^{-1}$ from the cloud velocity, this effect might lead to $15-20 \%$ uncertainties to the estimated outflow mass, and hence, to other calculated parameters. Together all these biases may lead to underestimate the estimated parameter up to a factor of $\sim 10$ even after correcting the estimated parameters for the mean inclination (Dunham et al. 2014). Table 1 lists the velocity range used to derive the calculations, $M_{\text {out }}$, and several other calculated dynamical parameters (i.e., $P_{\text {out }}, E_{\text {out }}, \dot{M}_{\text {out }}, F_{\text {out }}$, and $L_{\text {mech }}$ ); the values are listed for all the outflows after correcting for the inclination. As mentioned before, in this paper we estimated the outflow parameters based on the $\mathrm{CO}$ emission. So, in Table 1 we include only the outflows detected in $\mathrm{CO}$. The lobes identified in $\mathrm{HCN}$ or $\mathrm{HCO}^{+}$ but not detected in $\mathrm{CO}$ are not listed in the table. Additionally, in Table 1 we indicate whether the corresponding outflow is detected in either of the other two tracers. For reference, all the outflow lobes detected in all three tracers are marked in Figures 1 and A1-A4.

A histogram of $\dot{M}_{\text {out }}$ is shown in Figure 4 a. It can be seen that the values $\dot{M}_{\text {out }}$ have a wide range starting from $\sim 10^{-7}$ to $\sim 10^{-3}$ $M_{\odot} \mathrm{yr}^{-1}$. This provides us a unique opportunity to study the behavior of young outflows in a wide range of mass in Galactic massive protoclusters. Several authors reported that outflows associated with massive star formation are comparatively more powerful than those associated with low-mass star formation (Shepherd \& Churchwell 1996; Beuther et al. 2002; Wu et al. 2004; Hatchell et al. 2007; Yang et al. 2018; Li et al. 2018). While outflows with $M_{\text {out }}$ of $0.1-1 M_{\odot}$ and $\dot{M}_{\text {out }}$ of $10^{-7}-10^{-6} M_{\odot} \mathrm{yr}^{-1}$ are known as low-mass outflows, the outflows with $M_{\text {out }}$ of $10-1000 M_{\odot}$ and $\dot{M}_{\text {out }}$ of $10^{-5}-10^{-3} M_{\odot} \mathrm{yr}^{-1}$ are considered as high-mass outflows (Yang et al. 2018). Thus, a wide spread in the values of physical parameters for our observed outflows indicates that these correspond to low-mass to high-mass outflows. Note that the targets in this study are massive star-forming regions. We indeed found at least one massive outflow with $\dot{M}_{\text {out }}$ more than about $10^{-4} M_{\odot} \mathrm{yr}^{-1}$ in almost every region (except for I14382 and I17204).

A histogram for the dynamical age of the outflows are also shown in Figure 4b. The dynamical ages of the outflows in our sample are ranging from $0.1-2.8 \times 10^{4}$ years. These results confirm their driving sources to be young and still might be in an early phase when the matter is accreted onto the protostellar condensation.

\subsection{Mass of the host cores}

Smaller structures within molecular clouds are defined as clumps, cores, and substructures of a core based on their spatial extent in dust continuum and line emission maps. In this paper, we adopted the terminology recommended by Williams et al. (2000) and Wang et al. (2014): clumps have sizes $\sim 1 \mathrm{pc}$, dense cores have $\sim 0.1 \mathrm{pc}$, and substructures within cores have sizes $\sim 0.01 \mathrm{pc}$. Small scale structures in $0.9 \mathrm{~mm}$ continuum ALMA data were identified using the Python-based ASTRODENDRO-package ${ }^{2}$ which uses the DENDROGRAM algorithm to identify the hierarchical structures (i.e., cores) in the
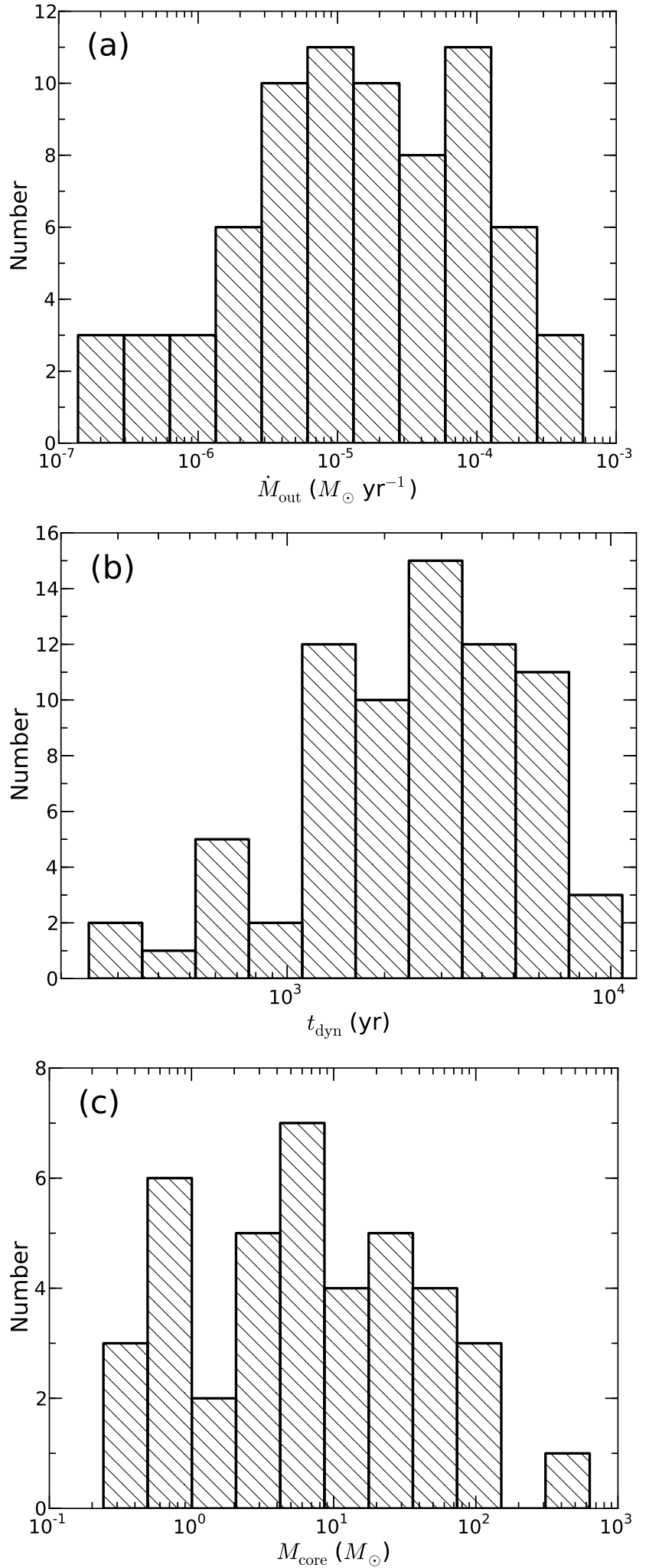

Figure 4. (a) Histogram of the outflow rate. (b) Histogram of the estimated dynamical ages of all the outflows, and (c) Histogram of the calculated mass of the host cores considering a dust temperature of $50 \mathrm{~K}$ and spectral index, $\beta$, of 2 .

\footnotetext{
2 https://dendrograms.readthedocs.io/en/stable/index.html
} 
Table 1. Outflow parameters (listed only those detected in $\mathrm{CO}$ ) derived from CO

\begin{tabular}{|c|c|c|c|c|c|c|c|c|c|c|c|c|c|c|c|c|c|c|c|}
\hline \multirow[t]{3}{*}{ Lobe Name } & $v(\mathrm{kn}$ & $\left.\mathrm{s}^{-1}\right)$ & & & $P_{\text {out }}$ & $10^{-1}$ & & & & & $\dot{M}_{\mathrm{ou}}$ & $10^{-5}$ & $F_{\text {out }}(1$ & $-4 M_{\odot}$ & & & & ed in & $\mathrm{wS}^{a}$ \\
\hline & & & $\left(10^{-}\right.$ & & $\mathrm{M}_{\odot} \mathrm{k}$ & & $\left(M_{\odot} \mathrm{k}\right.$ & $\mathrm{s}^{-2}$ & & & $\mathrm{M}_{\odot}$ & & $\mathrm{km} \mathrm{s}$ & $\mathrm{yr}^{-1}$ ) & & & HCN & $\mathrm{HCO}^{+}$ & \\
\hline & Blue & Red & Blue & Red & Blue & Red & Blue & Red & Blue & Red & Blue & Red & Blue & Red & Blue & Red & B R & B R & \\
\hline I14382_O1a & {$[-103,-65]$} & {$[-55,-14]$} & 0.5 & 0.2 & 1.8 & 0.6 & 4.2 & 1.5 & 0.9 & 0.5 & 0.5 & 0.3 & 2.0 & 1.2 & 3.9 & 2.3 & $\checkmark \checkmark$ & $x \times$ & $\mathrm{Y}$ \\
\hline I14382_Olb & - & {$[-55,-8]$} & - & 0.2 & - & 1.1 & - & 3.5 & - & 1.7 & - & 0.1 & - & 0.6 & - & 1.7 & $-\checkmark$ & $-x$ & $\mathrm{~N}$ \\
\hline I14382_O2 & {$[-129,-65]$} & {$[-55,6]$} & 1.0 & 0.5 & 3.7 & 2.2 & 10.3 & 6.3 & 0.6 & 0.6 & 1.7 & 0.8 & 6.1 & 3.7 & 14.1 & 8.7 & $\checkmark s$ & $\checkmark s$ & $\mathrm{Y}$ \\
\hline I14382_O3 & - & {$[-55,-32]$} & - & 0.1 & - & 0.2 & - & 0.4 & - & 1.7 & - & 0.0 & - & 0.1 & - & 0.2 & $-\checkmark$ & $-x$ & $\mathrm{~N}$ \\
\hline I14498_O1a & - & {$[-45,65]$} & - & 8.1 & - & 23.0 & - & 73.3 & - & 1.4 & - & 5.9 & - & 16.6 & - & 43.7 & $-\sqrt{ }$ & $-x$ & y \\
\hline I14498_O1b & {$[-112,-55]$} & - & 16.8 & - & 43.9 & - & 77.2 & - & 1.8 & - & 9.2 & - & 24.1 & - & 35.1 & - & $\checkmark-$ & $\sqrt{-}$ & y \\
\hline I14498_O2 & {$[-116,-55]$} & {$[-45,11]$} & 5.7 & 0.8 & 15.5 & 2.0 & 31.8 & 4.3 & 0.5 & 0.5 & 11.5 & 1.5 & 31.7 & 3.8 & 53.6 & 6.9 & $x \times$ & $x \times$ & $\mathrm{N}$ \\
\hline I14498_O3 & - & {$[-45,-34]$} & - & 2.4 & - & 3.5 & - & 2.8 & - & 6.9 & - & 0.3 & - & 0.5 & - & 0.3 & $-\checkmark$ & $-x$ & y \\
\hline I14498_O4 & - & {$[-45,-28]$} & - & 1.5 & - & 2.6 & - & 2.6 & - & 3.7 & - & 0.4 & - & 0.7 & - & 0.6 & $-x$ & $-x$ & y \\
\hline I15520_O1a & {$[-70,-46]$} & {$[-36,-16]$} & 0.9 & 5.8 & 2.6 & 11.4 & 4.3 & 13.0 & 4.0 & 7.0 & 0.2 & 0.8 & 0.7 & 1.6 & 0.9 & 1.5 & $\checkmark \checkmark$ & $\checkmark s$ & Y \\
\hline I15520_O2a & - & {$[-36,-2]$} & - & 0.1 & - & 0.4 & - & 0.9 & - & 4.2 & - & 0.0 & - & 0.1 & - & 0.2 & $-x$ & $-x$ & y \\
\hline I15520_O2b & {$[-70,-46]$} & - & 8.0 & - & 21.8 & - & 33.2 & - & 4.5 & - & 1.8 & - & 4.8 & - & 6.0 & - & $\checkmark-$ & $\sqrt{-}$ & y \\
\hline I15520_O3 & {$[-61,-46]$} & - & 2.0 & - & 4.3 & - & 4.9 & - & 7.3 & - & 0.3 & - & 0.6 & - & 0.6 & - & $\checkmark-$ & $\checkmark-$ & $\mathrm{N}$ \\
\hline I15520_O4 & {$[-99,-46]$} & - & 1.2 & - & 7.9 & - & 30.3 & - & 2.7 & - & 0.4 & - & 2.9 & - & 9.2 & - & $x-$ & $x-$ & $\mathrm{N}$ \\
\hline I15520_O5 & {$[-106,-46]$} & - & 3.0 & - & 11.6 & - & 32.4 & - & 1.8 & - & 1.7 & - & 6.6 & - & 15.2 & - & $\checkmark-$ & $\checkmark-$ & $\mathrm{N}$ \\
\hline I15596_O1a & {$[-119,-79]$} & {$[-69,-36]$} & 5.2 & 7.0 & 15.3 & 19.4 & 30.1 & 35.4 & 5.4 & 7.2 & 1.0 & 1.0 & 2.8 & 2.7 & 4.6 & 4.1 & $\checkmark s$ & $x \sqrt{x}$ & $\mathrm{~N}$ \\
\hline I15596_O1b & {$[-125,-79]$} & {$[-69,-44]$} & 6.7 & 2.2 & 18.6 & 4.8 & 36.0 & 6.5 & 2.9 & 3.7 & 2.3 & 0.6 & 6.5 & 1.3 & 10.4 & 1.4 & $\checkmark s$ & $x \sqrt{x}$ & $\mathrm{~N}$ \\
\hline I15596_O2a & {$[-122,-79]$} & {$[-69,10]$} & 3.6 & 5.1 & 10.5 & 18.4 & 20.6 & 59.4 & 0.8 & 0.6 & 4.4 & 9.1 & 13.0 & 32.8 & 21.0 & 87.8 & $\checkmark s$ & $\checkmark s$ & $\mathrm{~N}$ \\
\hline I15596_O2b & {$[-155,-79]$} & {$[-69,-5]$} & 1.6 & 3.0 & 7.0 & 9.5 & 24.9 & 26.1 & 0.3 & 0.4 & 5.9 & 7.6 & 25.6 & 24.4 & 75.3 & 55.7 & $\checkmark \checkmark$ & $\checkmark s$ & $\mathrm{~N}$ \\
\hline I15596_O3 & - & {$[-69,-22]$} & - & 1.7 & - & 5.5 & - & 14.5 & - & 1.4 & - & 1.2 & - & 4.0 & - & 8.6 & $-x$ & $-x$ & y \\
\hline I15596_O4 & {$[-113,-79]$} & {$[-69,-28]$} & 3.4 & 1.1 & 9.3 & 2.7 & 16.6 & 5.5 & 3.0 & 1.6 & 1.1 & 0.7 & 3.1 & 1.8 & 4.6 & 2.9 & $\checkmark \checkmark$ & $\checkmark \checkmark$ & Y \\
\hline I15596_O5 & {$[-166,-79]$} & {$[-69,26]$} & 7.0 & 6.8 & 45.8 & 51.0 & 225.1 & 266.6 & 1.7 & 1.0 & 4.1 & 7.0 & 26.6 & 52.9 & 108.4 & 228.8 & $x \sqrt{ }$ & $x<$ & $\mathrm{Y}$ \\
\hline I15596_O6 & {$[-122,-79]$} & {$[-69,-5]$} & 3.8 & 0.5 & 9.6 & 3.2 & 16.6 & 14.4 & 1.8 & 1.3 & 2.1 & 0.4 & 5.3 & 2.5 & 7.6 & 9.2 & $\checkmark d$ & $\checkmark \checkmark$ & Y \\
\hline I15596_O7 & {$[-104,-79]$} & - & 0.0 & - & 0.1 & - & 0.3 & - & 2.4 & - & 0.0 & - & 0.1 & - & 0.1 & - & $x-$ & $x-$ & y \\
\hline I15596_O8 & {$[-153,-79]$} & - & 5.6 & - & 20.4 & - & 55.6 & - & 1.3 & - & 4.2 & - & 15.3 & - & 34.5 & - & $\checkmark-$ & $\checkmark-$ & y \\
\hline I16060_O1 & {$[-120,-97]$} & {$[-81,-52]$} & 63.3 & 35.4 & 208.2 & 120.8 & 373.7 & 227.1 & 4.1 & 7.6 & 15.6 & 4.6 & 51.3 & 15.8 & 76.3 & 24.6 & $\checkmark s$ & $s \checkmark$ & $\mathrm{N}$ \\
\hline I16060_O2 & {$[-136,-97]$} & - & 69.7 & - & 274.3 & - & 606.8 & - & 6.1 & - & 11.4 & - & 44.8 & - & 82.2 & - & $\checkmark-$ & $\checkmark-$ & $\mathrm{N}$ \\
\hline I16060_O3 & - & {$[-81,-53]$} & - & 3.3 & - & 9.3 & - & 15.2 & - & 6.7 & - & 0.5 & - & 1.4 & - & 1.9 & $-x$ & $-x$ & y \\
\hline I16060_O4 & - & {$[-81,-66]$} & - & 0.8 & - & 2.1 & - & 2.9 & - & 15.9 & - & 0.1 & - & 0.1 & - & 0.2 & $-x$ & $-x$ & y \\
\hline I16071_O1a & {$[-139,-94]$} & {$[-78,-19]$} & 3.9 & 18.1 & 19.3 & 63.8 & 56.4 & 155.7 & 4.3 & 5.1 & 0.9 & 3.5 & 4.5 & 12.5 & 10.8 & 25.2 & $\checkmark s$ & $s \checkmark$ & $\mathrm{N}$ \\
\hline I16071_O1b & {$[-118,-94]$} & {$[-78,-58]$} & 11.1 & 8.3 & 35.2 & 22.6 & 64.6 & 35.1 & 6.7 & 5.3 & 1.6 & 1.6 & 5.2 & 4.2 & 8.0 & 5.5 & $\checkmark s$ & $x 5$ & $\mathrm{~N}$ \\
\hline I16071_O1c & {$[-122,-94]$} & {$[-78,-37]$} & 4.1 & 12.4 & 11.3 & 36.2 & 18.8 & 64.1 & 2.5 & 2.5 & 1.6 & 4.9 & 4.5 & 14.3 & 6.2 & 21.0 & $\checkmark \checkmark$ & $\checkmark \checkmark$ & $\mathrm{Y}$ \\
\hline I16071_O1d & {$[-225,-94]$} & - & 40.0 & - & 313.4 & - & 2017.0 & - & 1.1 & - & 37.8 & - & 295.7 & - & 1576.3 & - & $x-$ & $x-$ & y \\
\hline I16071_Ole & {$[-117,-94]$} & - & 12.0 & - & 33.5 & - & 53.1 & - & 3.8 & - & 3.2 & - & 8.8 & - & 11.6 & - & $\checkmark-$ & $\checkmark-$ & y \\
\hline I16071_O2 & {$[-192,-94]$} & {$[-78,58]$} & 4.8 & 3.8 & 41.2 & 32.5 & 241.3 & 245.1 & 0.3 & 0.2 & 18.6 & 16.7 & 159.3 & 142.1 & 772.5 & 887.9 & $\checkmark \checkmark$ & $x<$ & Y \\
\hline I16071_O3 & {$[-111,-94]$} & {$[-78,-58]$} & 6.5 & 1.8 & 19.4 & 4.5 & 31.7 & 6.2 & 3.4 & 7.5 & 1.9 & 0.2 & 5.8 & 0.6 & 7.8 & 0.7 & $\checkmark s$ & $\checkmark \checkmark$ & Y \\
\hline I16071_O4 & {$[-102,-94]$} & - & 2.3 & - & 5.0 & - & 5.8 & - & 9.8 & - & 0.2 & - & 0.5 & - & 0.5 & - & $\checkmark-$ & $\checkmark-$ & y \\
\hline I16071_O5 & - & {$[-78,-58]$} & - & 1.2 & - & 3.3 & - & 5.3 & - & 3.7 & - & 0.3 & - & 0.9 & - & 1.2 & $-x$ & $-x$ & y \\
\hline I16071_O6a & {$[-177,-94]$} & {$[-78,-56]$} & 4.4 & 0.8 & 25.6 & 2.5 & 96.3 & 4.2 & 0.3 & 1.0 & 14.0 & 0.8 & 82.0 & 2.5 & 255.5 & 3.5 & $\checkmark \checkmark$ & $\checkmark s$ & $\mathrm{~N}$ \\
\hline I16071_O6b & {$[-148,-94]$} & {$[-78,-2]$} & 10.5 & 13.7 & 59.8 & 71.8 & 202.9 & 275.0 & 1.4 & 1.0 & 7.8 & 13.2 & 44.2 & 69.1 & 124.2 & 219.2 & $\checkmark s$ & $\checkmark s$ & $\mathrm{~N}$ \\
\hline I16071_O7 & {$[-173,-94]$} & - & 11.1 & - & 61.9 & - & 262.4 & - & 1.6 & - & 7.0 & - & 38.8 & - & 136.2 & - & $\checkmark-$ & $\sqrt{-}$ & y \\
\hline I16076_O1a & {$[-125,-97]$} & {$[-77,-5]$} & 4.3 & 6.3 & 16.1 & 37.9 & 33.2 & 155.3 & 1.2 & 1.0 & 3.5 & 6.2 & 13.3 & 37.8 & 22.7 & 128.3 & $\checkmark s$ & $\checkmark s$ & $\mathrm{~N}$ \\
\hline I16076_O1b & {$[-111,-97]$} & {$[-77,20]$} & 7.5 & 4.3 & 23.3 & 21.4 & 37.9 & 78.0 & 2.7 & 0.8 & 2.7 & 5.3 & 8.5 & 26.3 & 11.4 & 79.5 & $\checkmark s$ & $x \sqrt{x}$ & $\mathrm{~N}$ \\
\hline I16076_O1c & - & {$[-77,-22]$} & - & 5.7 & - & 25.7 & - & 75.7 & - & 1.5 & - & 3.9 & - & 17.4 & - & 42.4 & $-\checkmark$ & $-\checkmark$ & $\mathrm{N}$ \\
\hline I16076_O1d & {$[-171,-97]$} & {$[-77,-10]$} & 0.8 & 1.9 & 4.1 & 10.0 & 15.2 & 32.5 & 0.8 & 0.5 & 1.1 & 3.8 & 5.3 & 19.6 & 16.3 & 52.5 & $x \times$ & $x \times$ & $\mathrm{N}$ \\
\hline I16076_Ole & - & {$[-77,-55]$} & - & 0.5 & - & 1.4 & - & 2.3 & - & 3.2 & - & 0.1 & - & 0.4 & - & 0.6 & $-\checkmark$ & $-x$ & y \\
\hline I16076_O1f & - & {$[-77,-55]$} & - & 0.1 & - & 0.3 & - & 0.5 & - & 3.8 & - & 0.0 & - & 0.1 & - & 0.1 & $-x$ & $-x$ & y \\
\hline I16076_O1g & {$[-147,-97]$} & {$[-77,-55]$} & 1.8 & 1.5 & 7.8 & 4.3 & 21.9 & 6.5 & 1.0 & 3.6 & 1.8 & 0.4 & 8.0 & 1.2 & 18.6 & 1.5 & $\checkmark \checkmark$ & $\checkmark s$ & $\mathrm{~N}$ \\
\hline I16076_Olh & {$[-115,-97]$} & {$[-77,-58]$} & 1.2 & 0.5 & 3.6 & 1.4 & 5.6 & 2.1 & 4.1 & 3.3 & 0.3 & 0.1 & 0.9 & 0.4 & 1.1 & 0.5 & $\sqrt{x}$ & $x \times$ & $\mathrm{Y}$ \\
\hline I16076_Oli & {$[-140,-97]$} & - & 2.1 & - & 10.2 & - & 28.7 & - & 2.2 & - & 1.0 & - & 4.7 & - & 10.9 & - & $\checkmark-$ & $\checkmark-$ & $\mathrm{N}$ \\
\hline I16076_O1j & {$[-116,-97]$} & - & 2.6 & - & 9.1 & - & 17.1 & - & 3.2 & - & 0.8 & - & 2.8 & - & 4.4 & - & $x-$ & $x-$ & $\mathrm{N}$ \\
\hline I16076_O1k & {$[-136,-97]$} & - & 0.9 & - & 2.9 & - & 5.6 & - & 1.3 & - & 0.7 & - & 2.2 & - & 3.6 & - & $x-$ & $x-$ & $\mathrm{N}$ \\
\hline I16076_O11 & {$[-119,-97]$} & - & 0.2 & - & 0.8 & - & 1.5 & - & 2.3 & - & 0.1 & - & 0.3 & - & 0.6 & - & $\checkmark-$ & $x-$ & $\mathrm{N}$ \\
\hline I16076_O2 & - & {$[-77,-55]$} & - & 3.4 & - & 10.8 & - & 18.6 & - & 7.2 & - & 0.5 & - & 1.5 & - & 2.1 & $\checkmark$ & $-x$ & y \\
\hline I16076_O3a & {$[-120,-97]$} & - & 3.2 & - & 9.3 & - & 14.9 & - & 5.2 & - & 0.6 & - & 1.8 & - & 2.4 & - & $x-$ & $x-$ & y \\
\hline I16076_O4a & - & {$[-77,-54]$} & - & 3.2 & - & 12.2 & - & 25.3 & - & 2.7 & - & 1.2 & - & 4.6 & - & 7.9 & $-x$ & $-x$ & $\mathrm{~N}$ \\
\hline I16076_O4b & {$[-121,-97]$} & - & 0.4 & - & 1.4 & - & 2.8 & - & 1.3 & - & 0.3 & - & 1.1 & - & 1.8 & - & $x-$ & $x-$ & $\mathrm{N}$ \\
\hline I16076_O5 & {$[-107,-97]$} & - & 0.2 & - & 0.7 & - & 1.0 & - & 4.5 & - & 0.1 & - & 0.1 & - & 0.2 & - & $x-$ & $x-$ & y \\
\hline I16272_O1a & {$[-84,-54]$} & {$[-38,18]$} & 13.1 & 15.6 & 40.3 & 62.8 & 72.5 & 173.7 & 3.2 & 2.4 & 4.1 & 6.6 & 12.7 & 26.6 & 18.9 & 60.9 & $\checkmark s$ & $\checkmark s$ & $\mathrm{Y}$ \\
\hline I16272_Olb & {$[-63,-54]$} & {$[-38,-27]$} & 0.8 & 0.5 & 1.7 & 1.1 & 1.9 & 1.3 & 11.1 & 7.0 & 0.1 & 0.1 & 0.2 & 0.2 & 0.1 & 0.2 & $\checkmark s$ & $\checkmark s$ & Y \\
\hline I16272_O1c & - & {$[-38,-18]$} & - & 0.2 & - & 0.7 & - & 1.2 & - & 2.4 & - & 0.1 & - & 0.3 & - & 0.4 & $\checkmark$ & $-x$ & y \\
\hline I16272_O2 & - & {$[-38,-2]$} & - & 3.2 & - & 9.3 & - & 15.9 & - & 2.4 & - & 1.3 & - & 3.8 & - & 5.4 & $-x$ & $-x$ & y \\
\hline I16351_O1a & {$[-58,-45]$} & {$[-35,48]$} & 13.7 & 32.3 & 30.7 & 202.6 & 36.8 & 833.8 & 2.7 & 0.6 & 5.1 & 52.6 & 11.5 & 329.7 & 11.4 & 1124.3 & $\checkmark s$ & $s$ & $\mathrm{Y}$ \\
\hline I16351_O1b & {$[-72,-45]$} & - & 1.8 & - & 4.5 & - & 6.8 & - & 4.2 & - & 0.4 & - & 1.1 & - & 1.3 & - & $x-$ & $x-$ & y \\
\hline I17204_O1a & {$[-54,-25]$} & {$[-9,0]$} & 2.4 & 0.5 & 7.4 & 1.1 & 12.7 & 1.2 & 1.9 & 4.5 & 1.3 & 0.1 & 3.9 & 0.2 & 5.5 & 0.2 & $\checkmark s$ & $\checkmark s$ & $\mathrm{~N}$ \\
\hline I17204_Olb & {$[-46,-25]$} & - & 0.9 & - & 2.7 & - & 4.8 & - & 3.4 & - & 0.2 & - & 0.8 & - & 1.2 & - & $\checkmark-$ & $\checkmark-$ & $\mathrm{N}$ \\
\hline I17204_O1c & {$[-49,-25]$} & - & 2.0 & - & 7.3 & - & 14.6 & - & 3.1 & - & 0.6 & - & 2.3 & - & 3.9 & - & $\checkmark-$ & $\sqrt{-}$ & $\mathrm{N}$ \\
\hline I17204_O1d & {$[-38,-25]$} & - & 5.7 & - & 14.9 & - & 20.1 & - & 8.6 & - & 0.7 & - & 1.7 & - & 1.9 & - & $x-$ & $\checkmark-$ & y \\
\hline I17220_O1 & {$[-123,-102]$} & {$[-86,-69]$} & 2.4 & 3.0 & 7.2 & 8.8 & 12.1 & 14.0 & 4.2 & 4.3 & 0.6 & 0.7 & 1.7 & 2.0 & 2.4 & 2.7 & $\checkmark s$ & $\checkmark s$ & $\mathrm{Y}$ \\
\hline I17220_O2 & {$[-140,-102]$} & {$[-86,0]$} & 11.6 & 10.2 & 38.5 & 60.7 & 80.1 & 286.0 & 3.5 & 1.7 & 3.3 & 6.0 & 10.8 & 35.7 & 18.7 & 139.2 & $\checkmark s$ & $x \times$ & $\mathrm{Y}$ \\
\hline I17220_O3 & {$[-146,-102]$} & {$[-86,-62]$} & 32.8 & 1.4 & 114.8 & 4.0 & 249.7 & 6.8 & 3.0 & 5.6 & 11.0 & 0.3 & 38.6 & 0.7 & 69.5 & 1.0 & $\checkmark \checkmark$ & $\checkmark x$ & Y \\
\hline I17220_O4 & {$[-143,-102]$} & {$[-86,-64]$} & 15.9 & 2.6 & 58.6 & 6.6 & 135.2 & 9.6 & 2.9 & 2.6 & 5.5 & 1.0 & 20.5 & 2.5 & 39.1 & 3.0 & $\checkmark \checkmark$ & $\checkmark s$ & $\mathrm{Y}$ \\
\hline I17220_O5 & - & {$[-86,-67]$} & - & 0.4 & - & 1.2 & - & 1.9 & - & 1.6 & - & 0.3 & - & 0.7 & - & 1.0 & $-x$ & $-x$ & y \\
\hline I17220_O6 & - & {$[-86,-63]$} & - & 0.6 & - & 1.9 & - & 3.5 & - & 1.5 & - & 0.4 & - & 1.3 & - & 2.0 & $-\checkmark$ & $-\sqrt{ }$ & $\mathrm{y}$ \\
\hline
\end{tabular}

${ }^{a}$ WS imples well-separated outflows without contamination from nearby lobes; $\mathrm{Y}$ and $\mathrm{y}$ indicates the bipolar and unipolar outflows, respectively. 
continuum maps. A detailed discussion of the algorithm can be found in Rosolowsky et al. (2008). The AstrodENDRO-package needs the inputs - the minimum flux level and the minimum number of pixels to be considered. A minimum flux level of $3 \sigma$ was adopted as a good value where $\sigma$ is the background flux estimated from the emissionfree areas in the continuum map. We considered structures having an area of more than the beam size of 25 pixels (corresponding to a spatial scale of $\sim 0.007-0.02 \mathrm{pc}$ at distances of 2.6-7.6 kpc) which is sufficient to identify dense cores. A detailed analysis of the cores will be presented in a future paper. In this paper, we only present the parameters (i.e., outflow name, coordinates of the continuum source, size, position angle, and integrated flux) of those 41 cores that are associated with outflows (see Table 2). These cores have typical sizes in the range from $0.01-0.06 \mathrm{pc}$ which confirms them to be cores or smaller substructures within cores. However, from now onward we shall refer to them as cores.

We further estimated the dust mass of each core assuming the dust emission to be optically thin using the following equation :

$M_{\text {dust }}=\frac{F_{\nu} d^{2}}{B_{v}\left(T_{\text {dust }}\right) \kappa_{v}}$,

where $F_{v}$ is the total dust continuum flux of the identified core at frequency $v, d$ is the distance to the source, $B_{v}\left(T_{\text {dust }}\right)$ is the Planck function at the temperature $T_{\text {dust }}$, and $\kappa_{v}=10\left(\frac{v}{1.2 T ~ H z}\right)^{\beta} \mathrm{cm}^{2} \mathrm{~g}^{-1}$ is the dust opacity (Hildebrand 1983) where $\beta$ is the spectral index. As mentioned before the targets here are massive star-forming regions and are associated with Hir regions. In such an environment, the cores in the vicinity of Hir regions could be externally heated to a high temperature of more than $100 \mathrm{~K}$ (see e.g., Osorio et al. 1999; Mookerjea et al. 2007, and references therein). Conversely, in the case of a cold star-forming core, $T_{\text {dust }}$ is typically assumed to be $\lesssim 30$ K (e.g., Plunkett et al. 2013; Wang et al. 2014). Hence, in the absence of a line-temperature measurement, we safely considered a dust temperature $\left(T_{\text {dust }}\right)$ of $50 \mathrm{~K}$ to calculate masses of the cores in these regions. The estimated mass of the cores for two dust emissivity spectral indices (i.e., $\beta=1.5$ and 2.0) are listed in Table 2 along with the other details of the identified cores. The number of outflow lobes associated with each core is also listed in the last column of Table 2. The mass estimated using $\beta=2.0$ is approximately a factor of 2 higher compared to the mass estimated using $\beta=1.5$.

A histogram for the core mass considering the $T_{\text {dust }}$ of $50 \mathrm{~K}$ and $\beta$ of 2, is shown in Figure 4c. The mass of the cores is typically up to a few tens of solar masses with a couple of sources more than one hundred solar masses. Note that the mass is estimated here assuming the emission to be optically thin which might not always hold. There is also a possibility that multiple cores are located along the line of sight, and together they may appear as a single core with considerably high mass. Such a possibility cannot be ignored given the fact that several cores in our sample drive multiple outflows (see Table 2), indicating the presence of multiple outflow driving sources within 0.1 pc scale. Hence, estimated core mass with such a large uncertainty may show a spurious relation when compared with the outflow parameters. To reduce such possibility, we defined a cleaned set of well-defined outflows (discussed in Section 4.2). This definition of well-defined outflows rejects the most massive cores in our sample which hold the highest possibility of having a companion. Although this precaution helps us to reduce contamination, we still cannot rule out the possibility of multiplicity in the identified cores as the regions are located considerably higher distances. There still might exist unresolved multiples. In an unresolved multiple, a single core may drive outflows while its companion(s) might remain inactive. Thus, the estimated core mass should be considered as an upper limit, and any derived relation or trend of outflow parameters with core masses should be treated with caution.

\section{DISCUSSION}

\subsection{Comparison among outflow parameters}

The regions studied here have shown a complex outflow morphology. Thus, estimated outflow parameters might be contamineted by the pixels of the nearby outflow lobes particularly toward the lower velocity ends. Thus, to separate out the contaminated lobes, we examined each region by overlaying the areas used to calculate the outflow parameters for all the identified lobes. Accordingly, we defined a cleaned set of well-separated outflow lobes which do not have any overlapping pixel (in the PP space) with a neighbouring lobe. The final column of Table 1 denotes whether the outflow lobe is well-separated or not. In Figure 5a, we have plotted $M_{\text {out }}$ versus $\dot{M}_{\text {out }}$ for all the lobes. The well-separated lobes are also marked in the figure. A nice correlation is noted between $\dot{M}_{\text {out }}$ and $M_{\text {out }}$ (Figure 5a) which is possibly expected as a higher gas outflow rate should inject more matter into the surrounding environments. Spearman's rank correlation coefficients $(\rho)$ are also marked in the figure for both the sets of data. We further plotted the $M_{\text {out }}$ with respect to the $t_{\text {dyn }}$ of all the lobes (Figure 5b). A non-correlation ( $\rho=0.07$ for all and $\rho$ $=-0.08$ for all well-separated lobes) can be noted among these two parameters. A similar non-correlation trend was noted previously by Wu et al. (2004) for the high-mass clumps. However, they noted an increasing trend of the outflow mass with time for the group of lowmass clumps. This is particularly puzzling for our sample because most of the outflows in this study are low-mass outflows. For further confirmation of this non-correlation, we performed a Student's $t$-test for the significance level of the null hypothesis following the similar method described in $\mathrm{Li}$ et al. (2003). We found that the test produces $p$-value greater than 0.9 which rejects the null hypothesis. This particular test indicates that the observed non-correlation might be a random result. A possible reason for this could be the comparatively smaller range of dynamical time-scales $\left(\sim 0.3-11 \times 10^{3} \mathrm{yr}\right)$ in our sample compared to that reported by Wu et al. $\left(2004, \sim 1-550 \times 10^{3}\right.$ yr).

We further examined the outflow rate against the dynamical timescale. An anti-correlation trend ( $\rho=-0.34$ and -0.42 for all and wellseparated lobes, respectively) is noted for $\dot{M}_{\text {out }}$ with $t_{\text {dyn }}$ (Figure 5c). This observed trend could be due to a decrease in the outflow rate with time as also previously suggested by several authors (Motte et al. 2007; López-Sepulcre et al. 2011; Watson et al. 2016, and references therein).

Figure $5 \mathrm{~d}$ shows the relation between the maximum dynamical time-scale $\left(t_{\mathrm{dyn}, \max }\right)$ of the outflows associated with a single core and the core mass. A hint of positive correlation is found for $t_{\mathrm{dyn}, \max }$ with the core mass (Spearman's Rank correlation coefficient $\rho=$ 0.35 and 0.16 for all and well-separated lobes, respectively). A positive trend was o observed in young infrared dark clouds by Li et al. (2020). This positive trend might indicate for the massive cores to have comparatively longer outflow dynamical timescale than their low-mass counterparts. If it is generally assumed that the dynamical time-scale of outflows reflects the accretion time-scale of the cores, then this positive trend might also hint at the possibility for the massive cores to have longer accretion history than the low mass cores in these protoclusters as also suggested by Li et al. (2020). However, as already discussed before a few cores in our sample might not be well-resolved. The presence of an unresolved companion would overestimate the core mass. Thus, this observed increasing trend might 
Table 2. Details of the driving cores

\begin{tabular}{|c|c|c|c|c|c|c|c|c|}
\hline $\begin{array}{r}\text { Outflow } \\
\text { Name }\end{array}$ & $\begin{array}{l}\alpha(\mathrm{J} 2000) \\
(\mathrm{h} \mathrm{m} \mathrm{s})\end{array}$ & $\begin{array}{c}\delta(\mathrm{J} 2000) \\
\left({ }^{\circ}, \prime \prime\right)\end{array}$ & $\begin{array}{l}\text { Source Size } \\
\operatorname{pc} \times p c, P A\left({ }^{\circ}\right)\end{array}$ & $\begin{array}{r}F_{\text {int }} \\
(\mathrm{mJy})\end{array}$ & $\begin{array}{c}M_{\text {Core }, 5} \\
\beta=1.5\end{array}$ & $\begin{array}{r}\mathrm{K}\left(M_{\odot}\right) \\
\beta=2.0\end{array}$ & $\begin{array}{l}\text { No. of } \\
\text { lobes }\end{array}$ & $\begin{array}{l}\text { Well-Defined } \\
\text { Outflow? }\end{array}$ \\
\hline I14382_O1 & 144202.106 & -603044.590 & $0.05 \times 0.03,89$ & 416.7 & 14.0 & 26.2 & 3 & $\mathrm{~N}$ \\
\hline I14382_O2 & 144203.066 & -603026.020 & $0.01 \times 0.01,163$ & 34.4 & 1.2 & 2.2 & 2 & $\mathrm{Y}$ \\
\hline I14382_O3 & 144202.833 & -603049.990 & $0.01 \times 0.01,132$ & 3.9 & 0.1 & 0.2 & 1 & $\mathrm{~N}$ \\
\hline I14498_O1 & 145342.681 & -590852.880 & $0.02 \times 0.01,177$ & 1034.7 & 21.2 & 39.6 & 2 & $\mathrm{Y}$ \\
\hline I14498_O2 & $* *$ & $* *$ & - & - & - & - & 2 & $\mathrm{~N}$ \\
\hline I14498_O3 & 145343.579 & -590843.780 & $0.04 \times 0.02,-135$ & 49.0 & 1.0 & 1.9 & 1 & $\mathrm{Y}$ \\
\hline I14498_O4 & 145342.941 & -590900.870 & $0.03 \times 0.02,87$ & 164.6 & 3.4 & 6.3 & 1 & $\mathrm{Y}$ \\
\hline I15520_O1 & 155548.398 & -524306.530 & $0.01 \times 0.00,-158$ & 133.1 & 1.8 & 3.4 & 2 & $\mathrm{Y}$ \\
\hline I15520_O2 & 155548.654 & -524308.660 & $0.01 \times 0.01,172$ & 433.4 & 5.9 & 10.9 & 2 & $\mathrm{~N}$ \\
\hline I15520_O3 & 155548.393 & -524304.380 & $0.02 \times 0.01,76$ & 1102.8 & 14.9 & 27.8 & 1 & $\mathrm{~N}$ \\
\hline I15520_O4 & 155548.848 & -524301.610 & $0.01 \times 0.00,98$ & 22.9 & 0.3 & 0.6 & 1 & $\mathrm{~N}$ \\
\hline I15520_O5 & 155549.265 & -524303.020 & $0.01 \times 0.01,-146$ & 21.2 & 0.3 & 0.5 & 1 & $\mathrm{~N}$ \\
\hline I15596_O1 & 160331.921 & -530922.960 & $0.01 \times 0.01,47$ & 63.3 & 2.5 & 4.6 & 4 & $\mathrm{~N}$ \\
\hline I15596_O2 & 160332.646 & -530926.820 & $0.02 \times 0.01,156$ & 108.5 & 4.2 & 7.8 & 4 & $\mathrm{~N}$ \\
\hline I15596_O3 & 160332.656 & -530945.760 & $0.03 \times 0.01,70$ & 12.4 & 0.5 & 0.9 & 1 & $\mathrm{Y}$ \\
\hline I15596_O4 & 160332.705 & -530929.570 & $0.02 \times 0.02,63$ & 86.1 & 3.3 & 6.2 & 2 & $\mathrm{Y}$ \\
\hline I15596_O5 & 160331.697 & -530932.090 & $0.03 \times 0.02,-176$ & 253.7 & 9.8 & 18.3 & 2 & $\mathrm{Y}$ \\
\hline I15596_O6 & $* *$ & ** & - & - & - & - & 2 & $\mathrm{Y}$ \\
\hline I15596_O7 & 160332.927 & -530927.850 & $0.01 \times 0.01,-155$ & 3.8 & 0.1 & 0.3 & 1 & $\mathrm{Y}$ \\
\hline I15596_O8 & 160330.635 & -530933.990 & $0.02 \times 0.01,159$ & 28.9 & 1.1 & 2.1 & 1 & $\mathrm{Y}$ \\
\hline I16060_O1 & 160952.650 & -515454.860 & $0.01 \times 0.01,165$ & 1205.3 & 65.3 & 121.7 & 2 & $\mathrm{~N}$ \\
\hline I16060_O2 & 160952.450 & -515455.790 & $0.02 \times 0.02,72$ & 3157.7 & 171.0 & 318.9 & 1 & $\mathrm{~N}$ \\
\hline I16060_O3 & $* *$ & $* *$ & - & - & - & - & 1 & $\mathrm{Y}$ \\
\hline I16060_O4 & 160952.803 & -515457.900 & $0.01 \times 0.01,-170$ & 143.1 & 7.8 & 14.4 & 1 & $\mathrm{Y}$ \\
\hline I16071_O1 & 161059.750 & -515023.540 & $0.06 \times 0.04,106$ & 7058.5 & 339.3 & 632.9 & 8 & $\mathrm{~N}$ \\
\hline I16071_O2 & 161059.553 & -515027.510 & $0.02 \times 0.01,53$ & 53.7 & 2.6 & 4.8 & 2 & $\mathrm{Y}$ \\
\hline I16071_O3 & 161059.400 & -515016.520 & $0.03 \times 0.02,160$ & 98.2 & 4.7 & 8.8 & 2 & $\mathrm{Y}$ \\
\hline I16071_O4 & 161100.242 & -515026.220 & $0.01 \times 0.01,-170$ & 5.2 & 0.2 & 0.5 & 1 & $\mathrm{Y}$ \\
\hline I16071_O5 & 161058.732 & -515036.370 & $0.04 \times 0.01,175$ & 29.2 & 1.4 & 2.6 & 1 & $\mathrm{Y}$ \\
\hline I16071_O6 & 161059.286 & -515011.780 & $0.01 \times 0.01,71$ & 5.6 & 0.3 & 0.5 & 4 & $\mathrm{~N}$ \\
\hline I16071_O7 & 161058.742 & -515017.330 & $0.02 \times 0.01,151$ & 10.0 & 0.5 & 0.9 & 1 & $\mathrm{Y}$ \\
\hline I16076_O1 & 161126.540 & -514157.320 & $0.04 \times 0.02,101$ & 858.8 & 43.0 & 80.2 & 17 & $\mathrm{~N}$ \\
\hline I16076_O2 & 161127.384 & -514150.210 & $0.02 \times 0.01,50$ & 10.2 & 0.5 & 0.9 & 1 & $\mathrm{Y}$ \\
\hline I16076_O3 & 161127.697 & -514155.360 & $0.05 \times 0.02,153$ & 255.7 & 12.8 & 23.9 & 1 & $\mathrm{Y}$ \\
\hline I16076_O4 & 161126.876 & -514155.920 & $0.01 \times 0.01,122$ & 16.4 & 0.8 & 1.5 & 1 & $\mathrm{~N}$ \\
\hline I16076_O5 & $* *$ & ** & - & - & - & - & 1 & $\mathrm{Y}$ \\
\hline I16272_O1 & 163058.770 & -484353.890 & $0.01 \times 0.01,-168$ & 2288.4 & 46.9 & 87.5 & 5 & $\mathrm{~N}$ \\
\hline I16272_O2 & $* *$ & ** & - & - & - & - & 1 & $\mathrm{Y}$ \\
\hline I16351_O1 & 163850.501 & -472800.910 & $0.02 \times 0.01,109$ & 2000.8 & 33.7 & 62.8 & 3 & $\mathrm{~N}$ \\
\hline I17204_O1 & 172350.249 & -363859.660 & $0.03 \times 0.02,148$ & 698.2 & 11.8 & 21.9 & 5 & $\mathrm{~N}$ \\
\hline I17220_O1 & 172525.635 & -361235.120 & $0.04 \times 0.03,-144$ & 175.0 & 20.2 & 37.7 & 2 & Y \\
\hline I17220_O2 & 172524.796 & -361236.850 & $0.03 \times 0.01,64$ & 12.7 & 1.5 & 2.7 & 2 & $\mathrm{Y}$ \\
\hline I17220_O3 & 172524.357 & -361247.890 & $0.03 \times 0.01,160$ & 20.3 & 2.4 & 4.4 & 2 & $\mathrm{Y}$ \\
\hline I17220_O4 & 172524.453 & -361239.360 & $0.03 \times 0.02,-147$ & 47.8 & 5.5 & 10.3 & 2 & $\mathrm{Y}$ \\
\hline I17220_O5 & 172524.926 & -361243.440 & $0.02 \times 0.01,160$ & 29.8 & 3.4 & 6.4 & 1 & $\mathrm{Y}$ \\
\hline I17220_O6 & 172525.697 & -361239.480 & $0.06 \times 0.03,178$ & 170.9 & 19.8 & 36.9 & 1 & $\mathrm{Y}$ \\
\hline
\end{tabular}

** The outflows without any detected continuum source

vary depending on an improved core mass estimation. For a more robust explanation, it needs a comparison of $t_{\text {dyn }}$ with the bolometric luminosity of the associated cores. However, the determination of bolometric luminosity requires multi-band observations of the source at a similar spatial resolution. No other observations are available for our targets at the angular resolution of ALMA, and it is thus difficult to determine the bolometric luminosity of the cores in our sample.

\subsection{Comparison between outflow parameters and core parameters}

Figure 6a shows the outflow mass plotted as a function of core mass,

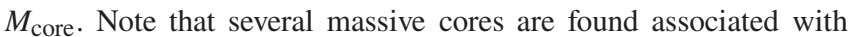
multiple outflows (both bipolar and unipolar). These outflows may be driven by further small-scale cores or multiple cores along the line of sight. However, such individual driving cores/sub-cores are not resolved even at the resolution of our ALMA data, possibly because our targets are densely clustered environment located at comparatively large distances $(2.6-7.6 \mathrm{kpc})$. Note that the presence of several outflows has been noted before in other massive star-forming regions (see e.g., Beuther et al. 2002; Zhang et al. 2007; Fernández-López et al. 

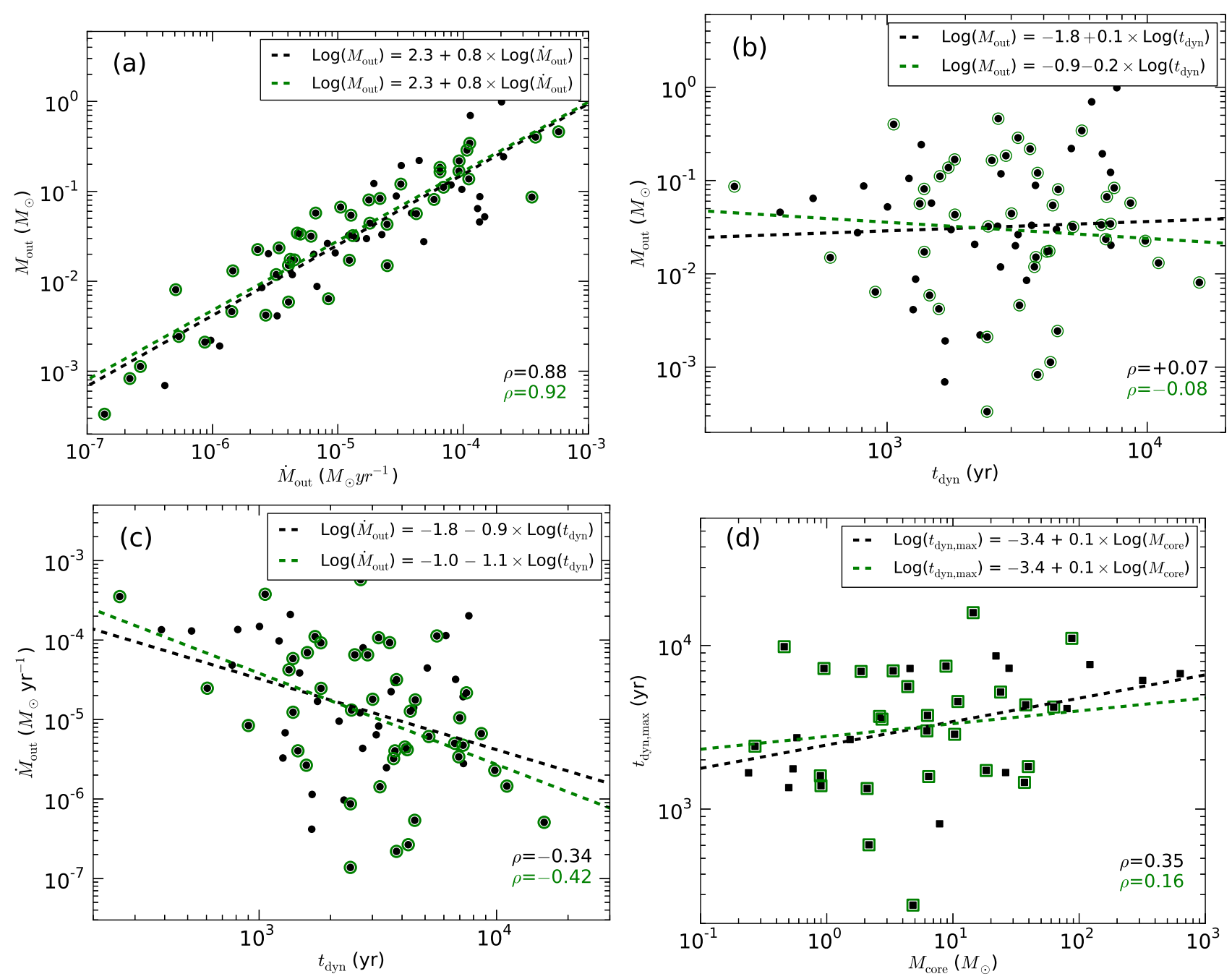

Figure 5. (a) Outflow mass versus the outflow rate for each lobe, (b) outflow mass against the dynamical time-scale of the outflows, (c) outflow rate against the dynamical time-scale of the outflows, and (d) the dynamical time-scale versus the mass of their host cores. The data points marked with green circles/square are for well-separated lobes. The black dashed lines in all the panels are the linear least-square fits on a logarithmic scale to all data points while the green dashed lines show the linear least-square fit to the well-separated data points in logarithminc scale. In each panel, the corresponing Spearman's Rank correlation coefficients $(\rho)$ are also written in black and green, respectively.

2013; Nony et al. 2020). In such a dense clustered environment, it is difficult to always identify bipolar signatures of outflows. For uniformity, we considered plotting the total outflow parameters (see triangles in Figure 6) driven by each identified core as that helps us to have a better comparison between the outflow and core properties. Additionally, for clarity we defined a cleaned well-defined set of outflow and their host cores. In Section 4.1, we already defined a set of well-separated lobes. Here, we further examine the well-separated outflows with respect to their host cores, and rejected those for which a host core is associated with more than one outflow (could be bipolar or unipolar). Finally, we named this cleaned set of data as well-defined outflows. We also marked these well-defined outflows in Figure 6 (see green triangles). Note that our calculated parameters are based on the emission detected in interferometric data which might lose the extended emission flux from the target. There are other additional components (i.e., assumption of optically thin emission, projection effect of outflow lobes, overlapping outflows) which underestimate the calculated parameters (see Section 3.3). Similarly, values of the cores might have a large uncertainty depend- ing on the adopted dust temperature and the spectral index in the calculation, missing extended emission, and presence of unresolved companions (see Section 3.4).

A correlation trend $(\rho=0.48)$ is found between the outflow mass and the core mass for all the outflows (Figure 6a). Even though an increasing trend is noted, a low correlation coefficient $(\rho=0.20)$ is obtained when only well-defined outflows are considered. The general increasing trend fits to a power-law of $M_{\text {out }} \propto M_{\text {core }}^{0.5}$ and $M_{\text {core }}^{0.4}$ for all and well-defined cases, respectively. A similar proportionality is seen at clump-scale by Yang et al. (2018, $M_{\text {out }} \propto M_{\text {clump }}^{0.6}$ ) for massive outflows associated with hundreds of ATLASGAL clumps. However, a few other studies found a slightly steeper relation between outflow mass and the core mass (i.e., $M_{\text {out }} \propto M_{\text {core }}^{0.8}$; Beuther et al. 2002; López-Sepulcre et al. 2009; de Villiers et al. 2014). Most of the previous studies were based on a few tens of outflows. Thus, the slightly shallower slope in our sample could be a result of a large range of core mass as also pointed out by Yang et al. (2018). The ratio $M_{\text {out }} / M_{\text {core }}$ has an average value of $0.05 \pm 0.13$ which implies that on average $5 \%$ of the core gas is entrained in the molecular outflow. 
This entrainment ratio is comparable to the value of 4-6\% reported by Beuther et al. (2002), Yang et al. (2018), and Li et al. (2018) seen at the clump-scale analysis.

Figure $6 \mathrm{~b}$ shows $\dot{M}_{\text {out }}$ plotted as a function of $M_{\text {core }}$. Despite a large scatter in the data points, a positive trend ( $\rho=0.42$ for all outflows and $\rho=0.24$ for well-defined outflows) can be noted for the wide-range of $M_{\text {core }}$ values. A similar positive trend with a similar slope was noted in previous studies toward massive clump-scale outflows (see López-Sepulcre et al. 2010; Yang et al. 2018; Li et al. 2018, and references therein). In addition, we also plotted outflow energy $\left(E_{\text {out }}\right)$ and mechanical luminosity of the outflow $\left(L_{\text {mech }}\right)$ with respect to the core mass, $M_{\text {core }}$ (see Figure $6 \mathrm{c}, \mathrm{d}$ ). A positive trend is noted for $E_{\text {out }}$ with $M_{\text {core }}$ for all outflows. However, the trend is not significant when only well-defined set of outflows are considered. Similarly, the positive trend for $L_{\text {mech }}$ with $M_{\text {core }}$ is not significant, specifically for the well-defined outflows. The correlation here is not statistically significant for both well-defined and all outflows (i.e. weak or non-correlation). Overall, these results suggest that the energy of an outflow generally increases with increasing mass of the driving core, but without a tight correlation.

The mechanical force of an outflow, $F_{\text {out }}$, can be considered as a measure of the strength of the outflow, and the rate at which the outflow injects momentum (at the current epoch) into the surrounding gas (Bachiller \& Gomez-Gonzalez 1992; Downes \& Cabrit 2007). Several studies have already reported a positive correlation of $F_{\text {out }}$ with core/clump mass and also with the bolometric luminosities (see Shepherd \& Churchwell 1996; Wu et al. 2004; Zhang et al. 2005; Yang et al. 2018, for example). Our calculated $F_{\text {out }}$ (mainly composed of low-mass outflows) is plotted against $M_{\text {core }}$ in Figure $7 \mathrm{a}$. An increasing trend ( $\rho=0.39$ and 0.21 for all and well-defined outflows, respectively) is noted for $F_{\text {out }}$ against core mass over 3 orders of magnitude. A linear fit to the data points shows a slope of 0.5 for all outflows and 0.4 for the well-defined outflows.

To examine the overall clump-scale scenario, we plotted the total value of $F_{\text {out }}$ for each region against the total mass of the clump (obtained from Liu et al. 2020a) along with the parameters for more than one hundred of ATLASGAL clumps studied by Yang et al. (2018) (see Figure 7b). In the absence of bolometric luminosity of individual cores, we also plotted the total $F_{\text {out }}$ of each target against the bolometric luminosity of the whole clump (see Urquhart et al. 2018; Liu et al. 2020a, for detailed parameters) along with parameters from Yang et al. (2018) (see Figure 7c). As can be seen in Figure 7b,c, our sample typically follows a similar increasing trend that was seen by Yang et al. (2018). The data points for our eleven targets roughly fall within the parametric distribution of Yang et al. (2018).

A hint of increasing trend for $F_{\text {out }}$ with $M_{\text {core }}$ that holds for 3 orders of magnitude of core mass indicates a possible similar mechanism for driving outflows over the whole range of low-mass to massive cores. However, a slightly different slope is obtained at core-scale (slope $=0.5$ and 0.4 , Figure 7a) compared to clump-scale (slope $=0.8$, Figure $7 \mathrm{~b}$ ). A similar increasing trend was noted before for low-mass and massive clumps by Maud et al. (2015) and Yang et al. (2018) for $F_{\text {out }}$ against bolometric luminosity. These authors, however, did not exclude the possibility of observational bias between the outflow force of low-luminosity and high-luminosity sources considering that they lie at different distances. The targets of this work are massive protoclusters where we primarily detected low-mass outflows along with a few high-mass outflows. We thus conclude that the increasing trend is possibly real as both low-mass and high-mass outflows in our study are identified in the same target region.

Considering the overall trend of the outflow parameters with the parametrs of the cores, a general inclination might be to believe that the stars of all different masses produce ouflows by a similar mechanism. However, as also discussed by Maud et al. (2015), the outflow parameters such as $\dot{M}_{\text {out }}$ and $F_{\text {out }}$ have an intrinsic dependency on the entrained mass by the outflows. Thus, these parameters can only infer the strength of the outflows, not the launching mechanism. The outflow launching mechanisms for low-mass and massive star formation could thus be substantially different.

The mass accretion rate could provide us the information on the ongoing star formation activity in the host cores. In general, an accretion rate of $\lesssim 10^{-5} \mathrm{M}_{\odot} \mathrm{yr}^{-1}$ is typical for low-mass star formation, whereas a much higher accretion rates of $\gtrsim 10^{-4} \mathrm{M}_{\odot} \mathrm{yr}^{-1}$ is required for massive star formation (McKee \& Tan 2003). It is possible to get an estimate of the accretion rate from the derived outflow mechanical force if it assumed that (1) the observed outflows are driven by protostellar winds from accretion disks (Keto 2003), (2) the momentum is conserved between the wind and the outflow, and (3) the wind and molecular gas interface is efficiently mixed (Richer et al. 2000). Then, the accretion rate can be formulated as $\dot{M}_{\text {acc }}=k \dot{M}_{\text {wind }}$, where $\dot{M}_{\text {wind }}$ is the mass-loss rate of the wind which can be inferred from the $F_{\text {out }}\left(=\dot{M}_{\text {wind }} v_{\text {wind }}\right)$. Here, $v_{\text {wind }}$ is the wind velocity which we adopted to be $500 \mathrm{~km} \mathrm{~s}^{-1}$ (Bally 2016; Li et al. 2020) and $k$ is the ratio between the mass accretion rate and the mass ejection rate which we assumed to be 3 (Shu et al. 2000). Accordingly, the derived accretion rates range from $4.6 \times 10^{-8}$ to $3.0 \times 10^{-4} \mathrm{M}_{\odot} \mathrm{yr}^{-1}$, with an average value of $2.4 \times 10^{-5} \mathrm{M}_{\odot} \mathrm{yr}^{-1}$. Although the mass accretion rates derived here are typically small compared to the expected accretion rate $\left(10^{-4}-10^{-3} \mathrm{M}_{\odot} \mathrm{yr}^{-1}\right)$ for high-mass star formation (McKee \& Tan 2003; Wang et al. 2010), a couple of sources also show comparable accretion rates that are seen toward high-mass star-forming regions (Zhang et al. 2005; Qiu et al. 2009; Liu et al. 2016, 2017).

These typically low accretion rates of $\lesssim 10^{-5} \mathrm{M}_{\odot} \mathrm{yr}^{-1}$ imply that the majority of the outflows in our studied regions are associated with low-mass star formation (see e.g., Machida \& Hosokawa 2013; Frank et al. 2014; Lee 2020). In the case of low-mass star formation, it is observed that the accretion rate falls off with time at an approximate $t^{-1}$ law (Caratti o Garatti et al. 2012). Additionally, the mass accretion rates for low-mass stars depends on the mass $\left(\mathbf{M}_{*}\right)$ of the forming star (see Alcalá et al. 2014; Biazzo et al. 2019, and references therein). While the determination of the stellar mass and the evolutionary stages of the observed cores is beyond the scope of this paper, we also note that uncertainties in mass accretion rates are large, resulting from the uncertainties of outflow parameters and adopted assumptions. Although this calculation prone to large uncertainty, it may still provide us the information on the general star formation scenario in these studied regions. We found that currently these regions are mostly going through an active phase of low-mass star formation.

\subsection{Energy budgets of the clumps and dissipation rate}

It is important to examine the cumulative impact of the outflows on the dynamics of their host clouds. We thus investigated whether the identified outflows have sufficient energy to drive the turbulence that counteracts the gravitational collapse of the host cloud. Estimation of the gravitational binding energy $\left(E_{\text {grav }}=3 G M_{\text {cloud }}^{2} / 5 R_{\text {cloud }}\right.$, where $G$ is gravitational constant) requires the total mass and the radius of the clouds. These values were obtained from Urquhart et al. (2018) (also see Table A1 of Liu et al. (2020a)). The mass, radius, and the 

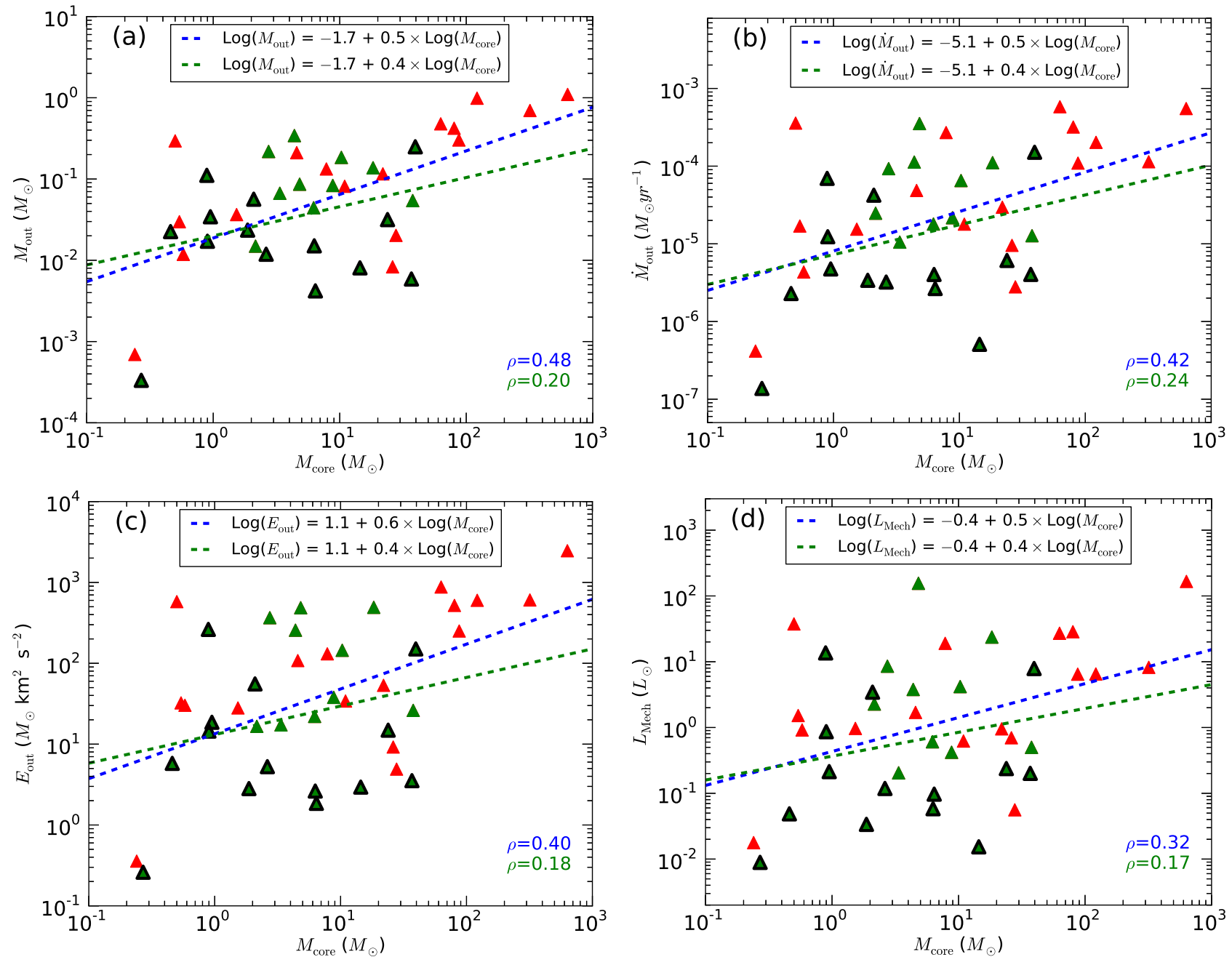

Figure 6. (a) The outflow mass versus the core mass, (b) the outflow rate versus the core mass, (c) the outflow energy versus the core mass, and (d) the mechanical luminosity of the outflow versus the core mass. The triangles represent the total outflow parameters associated with each core. The green triangles represent the well-defined outflows (see text). The green triangles with black edges correspond to the unipolar outflows while the remaining green triangles are for bipolar outflows. The red triangles are the sources that are contaminated either by driving multiple outflow lobes or the lobe has overlapping emission with a nearby lobe. The blue and green dashed lines are the linear least-square fit to all and green data points in the logarithmic scale, respectively. The Spearman's rank correlation coefficients ( $\rho$ in green for well-defined and in blue for all outflows) are also marked in all the panels.

Table 3. Energy budget of the observed protoclusters

\begin{tabular}{|c|c|c|c|c|c|c|c|c|c|c|}
\hline Source & $\begin{array}{r}\operatorname{Rad}^{a} \\
(\mathrm{pc})\end{array}$ & $\begin{array}{c}\log \left(L_{\mathrm{bol}}\right)^{a} \\
\left(L_{\odot}\right)\end{array}$ & $\begin{array}{c}\log \left(M_{\text {clump }}\right)^{a} \\
\left(M_{\odot}\right)\end{array}$ & $\begin{array}{c}\Delta v\left(\mathrm{C}^{17} \mathrm{O}\right)^{b} \\
\left(\mathrm{~km} \mathrm{~s}^{-1}\right)\end{array}$ & $E_{\text {grav }}$ & $\frac{E_{\text {turb }}}{\left(\times 10^{38}\right.}$ & $\frac{E_{\text {outflow }}}{)}$ & $\begin{array}{c}t_{\text {diss }} \\
\left(\times 10^{5} \mathrm{yr}\right)\end{array}$ & $\frac{L_{\text {turb }}}{\left(\times 10^{26}\right.}$ & $\frac{L_{\mathrm{Mech}}}{\left.J \mathrm{~s}^{-1}\right)}$ \\
\hline I14382-6017 & 1.68 & 5.2 & 3.6 & 3.03 & 484 & 197 & 1 & 6.4 & 10 & 17 \\
\hline I14498-5856 & 0.74 & 4.4 & 3.0 & 3.37 & 69 & 61 & 3 & 2.5 & 8 & 79 \\
\hline I15520-5234 & 0.67 & 5.1 & 3.2 & 4.53 & 192 & 175 & 2 & 1.7 & 33 & 19 \\
\hline I15596-5301 & 1.81 & 5.5 & 3.9 & 3.87 & 1789 & 640 & 13 & 5.4 & 38 & 373 \\
\hline I16060-5146 & 1.24 & 5.8 & 3.9 & 5.02 & 2612 & 1077 & 18 & 2.8 & 120 & 104 \\
\hline I16071-5142 & 1.21 & 4.8 & 3.7 & 5.80 & 1066 & 907 & 56 & 2.4 & 120 & 2286 \\
\hline I16076-5134 & 1.57 & 5.3 & 3.6 & 4.89 & 518 & 512 & 9 & 3.7 & 44 & 230 \\
\hline I16272-4837 & 0.84 & 4.3 & 3.2 & 3.66 & 154 & 114 & 4 & 2.6 & 14 & 48 \\
\hline I16351-4722 & 0.69 & 4.9 & 3.2 & 4.59 & 187 & 180 & 13 & 1.7 & 33 & 638 \\
\hline I17204-3636 & 0.60 & 4.2 & 2.9 & 3.11 & 54 & 41 & 1 & 2.2 & 6 & 7 \\
\hline I17220-3609 & 2.41 & 5.7 & 4.3 & 4.53 & 8479 & 2203 & 12 & 6.1 & 114 & 156 \\
\hline
\end{tabular}

${ }^{a}$ Values from Urquhart et al. (2018)

$b$ Values from Yue et al. (private communication) 

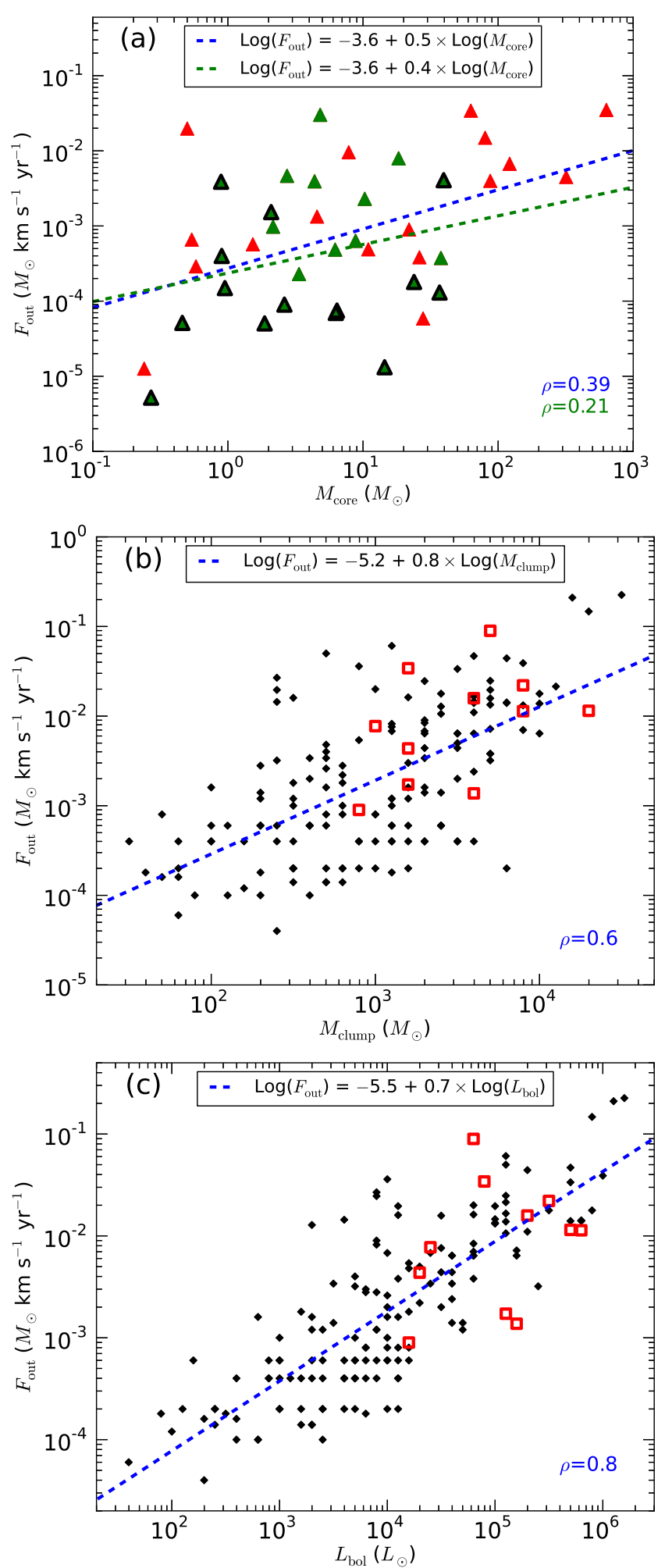

Figure 7. (a) Outflow force plotted against the mass of the associated cores. The triangles represent the total outflow parameters associated with each core. The green triangles represent the well-defined outflows whereas the red triangles are contaminated lobes (see text and caption of Figure 6). The blue and green dashed lines are the linear least-square fit to all and green data points in the logarithmic scale, respectively. (b) Total outflow force plotted against the mass of the whole clump. (c) Total outflow force plotted against the bolometric luminosity of the clump. Red squares in panels (b) and (c) show the data points for our targets while the black diamonds are obtained from Yang et al. (2018). Blue dashed lines in both the panels represent the linear least-square fits on a logarithmic scale. The equation of the best fit lines

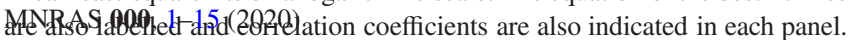
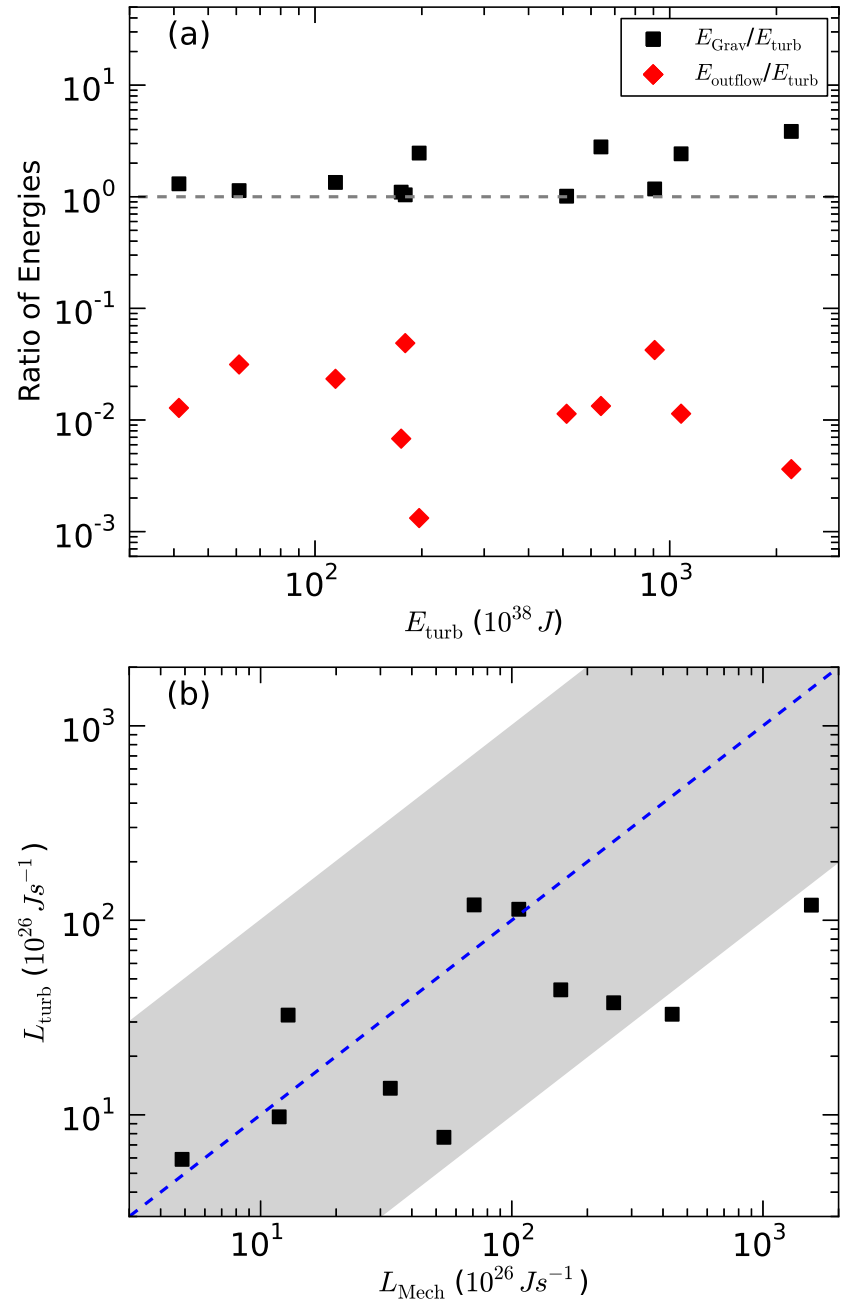

Figure 8. (a) The ratios $E_{\text {grav }} / E_{\text {turb }}$ (black squares) and $E_{\text {outflow }} / E_{\text {turb }}$ (red diamonds) plotted as a function of the turbulent energy. The dashed black line represents the ratio of one. (b) The comparison between turbulent dissipation rate and the mechanical energy injection rate. The blue dashed line represents equal rates of turbulent dissipation and mechanical energy injection, and the grey-shaded area shows a variation of one order of magnitude around the blue dashed line.

calculated values of $E_{\text {grav }}$ are listed in Table 3. The turbulent energy of a molecular cloud is given by

$E_{\text {turb }}=\frac{1}{2} M_{\text {cloud }} \sigma_{3 \mathrm{~d}}^{2}$,

where $\sigma_{3 \mathrm{~d}}$ is the three-dimensional turbulent velocity dispersion, which can be calculated using

$\sigma_{3 \mathrm{~d}}=\frac{\sqrt{3}}{2 \sqrt{2 \ln 2}} \Delta v_{\mathrm{FWHM}}$,

where $\Delta v_{\text {FWHM }}$ is the one-dimensional velocity width of an optically thin tracer of the molecular cloud. We obtained the $\Delta v_{\text {FWHM }}$ values for all our regions from Yue et al. (private communication). They performed the $\mathrm{C}^{17} \mathrm{O}$ (3-2) line observations of these sources using the Atacama Pathfinder Experiment (APEX) and obtained the $\Delta v_{\text {FWHM }}$ values by performing a Gaussian fit to the observed line profile. The values of $\Delta v_{\text {FWHM }}\left(\right.$ i.e., $\left.\Delta v\left(\mathrm{C}^{17} \mathrm{O}\right)\right)$ and the calculated $E_{\text {turb }}$ 
are listed in Table 3. The table also lists the total energy of all the outflows $\left(E_{\text {outflow }}\right)$ identified in each region. In Figure 8a we have shown the ratios of $E_{\text {grav }} / E_{\text {turb }}$ and $E_{\text {outflow }} / E_{\text {turb }}$ versus the turbulent energy. As can be seen in the figure, although $E_{\text {grav }}$ is generally comparable to $E_{\text {turb }}$, the total outflow energy, $E_{\text {outflow }}$, is typically 1-3 orders of magnitude smaller than the turbulent energies in these regions, and thus, cannot drive the turbulence in these protoclusters. A similar conclusion was reached for outflows in the Taurus molecular cloud by $\mathrm{Li}$ et al. (2015). However, the regions studied here are massive protoclusters and are mostly associated with the $\mathrm{H}$ in regions. Thus, a significant amount of turbulence might be contributed by the massive stars and the associated $\mathrm{H}$ II region. Note that the origin of such turbulence in the molecular clouds is unclear (see Larson 1981; Heyer \& Brunt 2004). Hennebelle \& Falgarone (2012) suggested that such turbulence could be driven by external sources, such as, supernovae explosion, large-scale Hi streams (Ballesteros-Paredes et al. 1999), shocks (McKee \& Ostriker 2007), or galactic differential rotation (Klessen \& Hennebelle 2010; Hennebelle \& Falgarone 2012).

We further estimated the total outflow energy injection rate (i.e., $L_{\mathrm{Mech}}$ ) and compare it with the energy rate needed to maintain the turbulence of the clump (i.e., $L_{\text {turb }}$ ). The turbulent dissipation rate can be calculated as

$L_{\text {turb }}=\frac{E_{\text {turb }}}{t_{\text {diss }}}$,

where $t_{\text {diss }}$ is the turbulent dissipation time. The turbulent dissipation time is calculated using the equation based on numerical simulations (McKee \& Ostriker 2007).

$t_{\text {diss }} \sim 0.5 \frac{D_{\text {cloud }}}{\sigma_{1 \mathrm{~d}}}$

where $D_{\text {cloud }}$ is the diameter of the clump and $\sigma_{1 \mathrm{~d}}$ is the onedimensional turbulent velocity dispersion along the line of sight (i.e., $\sigma_{1 \mathrm{~d}}=\frac{\sigma_{3 \mathrm{~d}}}{\sqrt{3}}$.

The calculated turbulence dissipation time, the turbulence energy dissipation rate, and the total outflow energy rate for all the targets in our sample are listed in Table 3. Note that these values should be treated only as order-of-magnitude estimates because there are several uncertainties involved in the estimated outflow parameters as already discussed in Section 3.3. In Figure 8b, the turbulent energy dissipation rate, $L_{\text {turb }}$, is plotted with respect to the total outflow energy injection rate, $L_{\mathrm{Mech}}$, for our eleven protoclusters. It can be seen in the figure that the $L_{\text {turb }}$ is typically comparable within an order of magnitude of the $L_{\mathrm{Mech}}$ values for all the clumps. Thus, it seems that the outflow observed here cannot account for the generation of turbulence, but can sustain the turbulence at the current epoch as it is similar to the estimated dissipation rate, similar to what was found in low mass star forming regions (e.g., Li et al. 2015).

\section{CONCLUSIONS}

We studied the properties of the outflows toward 11 Galactic massive protoclusters. Outflows are identified using three different tracers, the $\mathrm{CO}(3-2), \mathrm{HCN}(4-3)$, and $\mathrm{HCO}^{+}$(4-3) lines. A total of 106 outflow lobes have been detected in these regions as already reported in Paper I. About $40 \%$ of these outflows are identified with a single lobe, possibly because of the dense clustering nature of the massive protoclusters. All the physical parameters studied here are derived from $\mathrm{CO}$. The main findings of this study are the following.

- Although the position angles of outflow lobes do not differ in the three tracers, $\mathrm{HCN}$ and $\mathrm{HCO}^{+}$generally detect outflows at lower terminal velocities compared to $\mathrm{CO}$. This is possibly because $\mathrm{CO}$ is more sensitive to detect the low density and high-velocity outflowing material compared to the $\mathrm{HCN}$ and $\mathrm{HCO}^{+}$.

- Identified outflows are young (typical $t_{\text {dyn }} \sim 10^{2}-10^{4} \mathrm{yr}$ ) and are composed of low-mass to (a few) massive outflows (e.g., $\dot{M}_{\text {out }} \sim$ $\left.10^{-7}-10^{-4} M_{\odot} \mathrm{yr}^{-1} ; P_{\text {out }} \sim 10^{-3}-10^{+1} M_{\odot} \mathrm{km} \mathrm{s}^{-1}\right)$.

- An anti-correlation trend is noted for $\dot{M}_{\text {out }}$ versus $t_{\text {dyn }}$. This particular result may indicate that the outflow rate decreases with time. Although not significant, increase of $t_{\text {dyn }}$ with the mass of the associated core probably indicates that the massive cores might have longer accretion history than the low mass cores in these protoclusters.

- The outflow mass for our sample shows a trend that increase with core mass with a power-law index of $\sim 0.4-0.5$. This index is in agreement with clump-scale massive outflows reported by Yang et al. (2018), but shallower (power index 0.9) than found in a few previous findings (Beuther et al. 2002; López-Sepulcre et al. 2009; de Villiers et al. 2014; Li et al. 2018). A similar rising trendency is seen for $F_{\text {out }}$ with respect to core mass which could be a result of increasing entrained mass with increasing mass of the driving source, rather than the outflow launching mechanism.

- Calculation of the energy budget reveals that the kinetic energy of outflows alone cannot balance the gravitational binding energy, and is also unable to generate the observed turbulence in the host molecular clouds of our target regions. However, the energy injection rates of outflows in most of the regions can sustain the turbulence at the current epoch.

\section{ACKNOWLEDGEMENTS}

We thank an anonymous referee for the thorough review and critical comments that helped us to improve the scientific content and the presentation of the manuscript. The work is supported by National Science Foundation of China (11988101, 11721303, 11973013, U1631102, 11373010). TB and KW are supported by the National Key Research and Development Program of China (2017YFA0402702, 2019YFA0405100). KW also acknowledges the support from a starting grant at the Kavli Institute for Astronomy and Astrophysics, Peking University (7101502016). TB acknowledges funding from the China Postdoctoral Science Foundation through grant 2018M631241 and the PKU-Tokyo Partner fund. TB also acknowledge the support from S. N. Bose National Centre for Basic Sciences under the Department of Science and Technology (DST), Govt. of India. This work was carried out in part at the Jet Propulsion Laboratory, operated for NASA by the California Institute of Technology. L.B. acknowledges support from CONICYT project Basal AFB-170002. C.W.L. is supported by the Basic Science Research Program through the National Research Foundation of Korea (NRF) funded by the Ministry of Education, Science and Technology (NRF-2019R1A2C1010851). This research made use of Astropy, a community-developed core Python package for astronomy (Astropy Collaboration et al. 2018). This paper makes use of the following ALMA data: ADS/JAO.ALMA\#2017.1.00545.S. ALMA is a partnership of ESO (representing its member states), NSF (USA) and NINS (Japan), together with NRC (Canada), MOST and ASIAA (Taiwan), and KASI (Republic of Korea), in cooperation with the Republic of Chile. The Joint ALMA Observatory is operated by ESO, AUI/NRAO, and NAOJ. 


\section{DATA AVAILABILITY}

The raw data that support this study are publicly available for download from the ALMA archive. The reduced data are available from the corresponding author upon reasonable request.

\section{REFERENCES}

Alcalá J. M., et al., 2014, A\&A, 561, A2

Arce H. G., Goodman A. A., 2001, ApJ, 554, 132

Arce H. G., Shepherd D., Gueth F., Lee C. F., Bachiller R., Rosen A., Beuther H., 2007, in Reipurth B., Jewitt D., Keil K., eds, Protostars and Planets V. p. 245 (arXiv: astro-ph/0603071)

Arce H. G., Borkin M. A., Goodman A. A., Pineda J. E., Halle M. W., 2010, ApJ, 715, 1170

Arce H. G., Mardones D., Corder S. A., Garay G., Noriega-Crespo A., Raga A. C., 2013, ApJ, 774, 39

Astropy Collaboration et al., 2018, AJ, 156, 123

Bachiller R., 1996, ARA\&A, 34, 111

Bachiller R., Gomez-Gonzalez J., 1992, A\&ARv, 3, 257

Ballesteros-Paredes J., Hartmann L., Vázquez-Semadeni E., 1999, ApJ, 527,285

Bally J., 2016, ARA\&A, 54, 491

Baug T., et al., 2020, ApJ, 890, 44

Beuther H., Schilke P., Sridharan T. K., Menten K. M., Walmsley C. M., Wyrowski F., 2002, A\&A, 383, 892

Biazzo K., Beccari G., De Marchi G., Panagia N., 2019, ApJ, 875, 51

Blake G. A., Sutton E. C., Masson C. R., Phillips T. G., 1987, ApJ, 315, 621

Bonnell I. A., Bate M. R., Clarke C. J., Pringle J. E., 2001, MNRAS, 323, 785

Bontemps S., Andre P., Terebey S., Cabrit S., 1996, A\&A, 311, 858

Caratti o Garatti A., et al., 2012, A\&A, 538, A64

Caratti o Garatti A., Stecklum B., Linz H., Garcia Lopez R., Sanna A., 2015, A\&A, 573, A82

Carroll J. J., Frank A., Blackman E. G., Cunningham A. J., Quillen A. C., 2009, ApJ, 695, 1376

Downes T. P., Cabrit S., 2007, A\&A, 471, 873

Dunham M. M., Arce H. G., Mardones D., Lee J.-E., Matthews B. C., Stutz A. M., Williams J. P., 2014, ApJ, 783, 29

Feddersen J. R., et al., 2020, ApJ, 896, 11

Fernández-López M., Girart J. M., Curiel S., Zapata L. A., Fonfría J. P., Qiu K., 2013, ApJ, 778, 72

Franco J., 1983, ApJ, 264, 508

Frank A., et al., 2014, in Beuther H., Klessen R. S., Dullemond C. P., Henning T., eds, Protostars and Planets VI. p. 451 (arXiv: 1402.3553), doi:10.2458/azu_uapress_9780816531240-ch020

Green J. D., et al., 2011, ApJ, 731, L25

Hartmann L., Herczeg G., Calvet N., 2016, ARA\&A, 54, 135

Hatchell J., Fuller G. A., Richer J. S., 2007, A\&A, 472, 187

Hennebelle P., Falgarone E., 2012, A\&ARv, 20, 55

Heyer M. H., Brunt C. M., 2004, ApJ, 615, L45

Hildebrand R. H., 1983, QJRAS, 24, 267

Keto E., 2003, ApJ, 599, 1196

Kim K.-T., Kurtz S. E., 2006, ApJ, 643, 978

Klessen R. S., Hennebelle P., 2010, A\&A, 520, A17

Kwan J., Scoville N., 1976, ApJ, 210, L39

Lada C. J., 1985, ARA\&A, 23, 267

Larson R. B., 1981, MNRAS, 194, 809

Lee C.-F., 2020, A\&ARv, 28, 1

Lee C.-F., Mundy L. G., Reipurth B., Ostriker E. C., Stone J. M., 2000, ApJ, 542,925

Li D., Goldsmith P. F., Menten K., 2003, ApJ, 587, 262

Li H., et al., 2015, ApJS, 219, 20

Li Q., et al., 2018, ApJ, 867, 167

Li S., Zhang Q., Pillai T., Stephens I. W., Wang J., Li F., 2019, ApJ, 886, 130

Li S., et al., 2020, arXiv e-prints, p. arXiv:2009.05506

Liu T., et al., 2016, ApJ, 824, 31

Liu T., et al., 2017, ApJ, 849, 25
Liu T., et al., 2020a, MNRAS, 496, 2790

Liu T., et al., 2020b, MNRAS, 496, 2821

López-Sepulcre A., Codella C., Cesaroni R., Marcelino N., Walmsley C. M., 2009, A\&A, 499, 811

López-Sepulcre A., Cesaroni R., Walmsley C. M., 2010, A\&A, 517, A66

López-Sepulcre A., et al., 2011, A\&A, 526, L2

Machida M. N., Hosokawa T., 2013, MNRAS, 431, 1719

Mangum J. G., Shirley Y. L., 2015, PASP, 127, 266

Matzner C. D., Jumper P. H., 2015, ApJ, 815, 68

Maud L. T., Moore T. J. T., Lumsden S. L., Mottram J. C., Urquhart J. S., Hoare M. G., 2015, MNRAS, 453, 645

McKee C. F., Ostriker E. C., 2007, ARA\&A, 45, 565

McKee C. F., Tan J. C., 2003, ApJ, 585, 850

Molinari S., Testi L., Rodríguez L. F., Zhang Q., 2002, ApJ, 570, 758

Mookerjea B., Casper E., Mundy L. G., Looney L. W., 2007, ApJ, 659, 447

Motte F., Bontemps S., Schilke P., Schneider N., Menten K. M., Broguière D., 2007, A\&A, 476, 1243

Nakamura F., Li Z.-Y., 2007, ApJ, 662, 395

Nakamura F., et al., 2011, ApJ, 726, 46

Narayanan G., Snell R., Bemis A., 2012, MNRAS, 425, 2641

Nony T., et al., 2020, A\&A, 636, A38

Offner S. S. R., Lee E. J., Goodman A. A., Arce H., 2011, ApJ, 743, 91

Osorio M., Lizano S., D’Alessio P., 1999, ApJ, 525, 808

Plunkett A. L., Arce H. G., Corder S. A., Mardones D., Sargent A. I., Schnee S. L., 2013, ApJ, 774, 22

Qiu K., Zhang Q., Wu J., Chen H.-R., 2009, ApJ, 696, 66

Reiter M., Kiminki M. M., Smith N., Bally J., 2017, MNRAS, 470, 4671

Riaz B., Briceño C., Whelan E. T., Heathcote S., 2017, ApJ, 844, 47

Richer J. S., Shepherd D. S., Cabrit S., Bachiller R., Churchwell E., 2000, in Mannings V., Boss A. P., Russell S. S., eds, Protostars and Planets IV. p. 867 (arXiv: astro-ph/9904097)

Rosolowsky E. W., Pineda J. E., Kauffmann J., Goodman A. A., 2008, ApJ, 679, 1338

Shepherd D. S., Churchwell E., 1996, ApJ, 457, 267

Shu F. H., Adams F. C., Lizano S., 1987, ARA\&A, 25, 23

Shu F. H., Najita J. R., Shang H., Li Z. Y., 2000, in Mannings V., Boss A. P., Russell S. S., eds, Protostars and Planets IV. pp 789-814

Solomon P. M., Huguenin G. R., Scoville N. Z., 1981, ApJ, 245, L19

Takahashi S., Machida M. N., Tomisaka K., Ho P. T. P., Fomalont E. B., Nakanishi K., Girart J. M., 2019, ApJ, 872, 70

Tan J. C., Beltrán M. T., Caselli P., Fontani F., Fuente A., Krumholz M. R., McKee C. F., Stolte A., 2014, in Beuther H., Klessen R. S., Dullemond C. P., Henning T., eds, Protostars and Planets VI. p. 149 (arXiv: 1402 . 0919), doi:10.2458/azu_uapress_9780816531240-ch007

Urquhart J. S., et al., 2018, MNRAS, 473, 1059

Wang P., Li Z.-Y., Abel T., Nakamura F., 2010, ApJ, 709, 27

Wang K., Zhang Q., Wu Y., Zhang H., 2011, ApJ, 735, 64

Wang K., et al., 2014, MNRAS, 439, 3275

Watson D. M., et al., 2016, ApJ, 828, 52

Whelan E. T., Ray T. P., Bacciotti F., Natta A., Testi L., Randich S., 2005, Nature, 435, 652

Williams J. P., Blitz L., McKee C. F., 2000, in Mannings V., Boss A. P., Russell S. S., eds, Protostars and Planets IV. p. 97 (arXiv: astro-ph/9902246)

Wu Y., Wei Y., Zhao M., Shi Y., Yu W., Qin S., Huang M., 2004, A\&A, 426, 503

Yang A. Y., Thompson M. A., Urquhart J. S., Tian W. W., 2018, ApJS, 235, 3

Zapata L. A., Schmid-Burgk J., Muders D., Schilke P., Menten K., Guesten R., 2010, A\&A, 510, A2

Zhang Q., Hunter T. R., Brand J., Sridharan T. K., Cesaroni R., Molinari S., Wang J., Kramer M., 2005, ApJ, 625, 864

Zhang Q., Hunter T. R., Beuther H., Sridharan T. K., Liu S. Y., Su Y. N., Chen H. R., Chen Y., 2007, ApJ, 658, 1152

Zuckerman B., Kuiper T. B. H., Rodriguez Kuiper E. N., 1976, ApJ, 209, L137 de Villiers H. M., et al., 2014, MNRAS, 444, 566 
APPENDIX A: A. OUTFLOW LOBES FOR ALL THE REGIONS

As presented in Section 3.1, we identified the outflow lobes using three different tracers (i.e., $\mathrm{CO}, \mathrm{HCN}$, and $\mathrm{HCO}^{+}$). An example figure for $\mathrm{CO}$ and $\mathrm{HCN}$ lobes is presented in Figure 1. Figures corresponding to the rest of the regions with $\mathrm{HCN}$ lobes are presented in Figures A1 and A2. The figures with $\mathrm{HCO}^{+}$outflow lobes are also presented in Figures A3 and A4.

This paper has been typeset from a $\mathrm{T}_{\mathrm{E}} \mathrm{X} / \mathrm{L} \mathrm{T} \mathrm{E} \mathrm{X}$ file prepared by the author. 

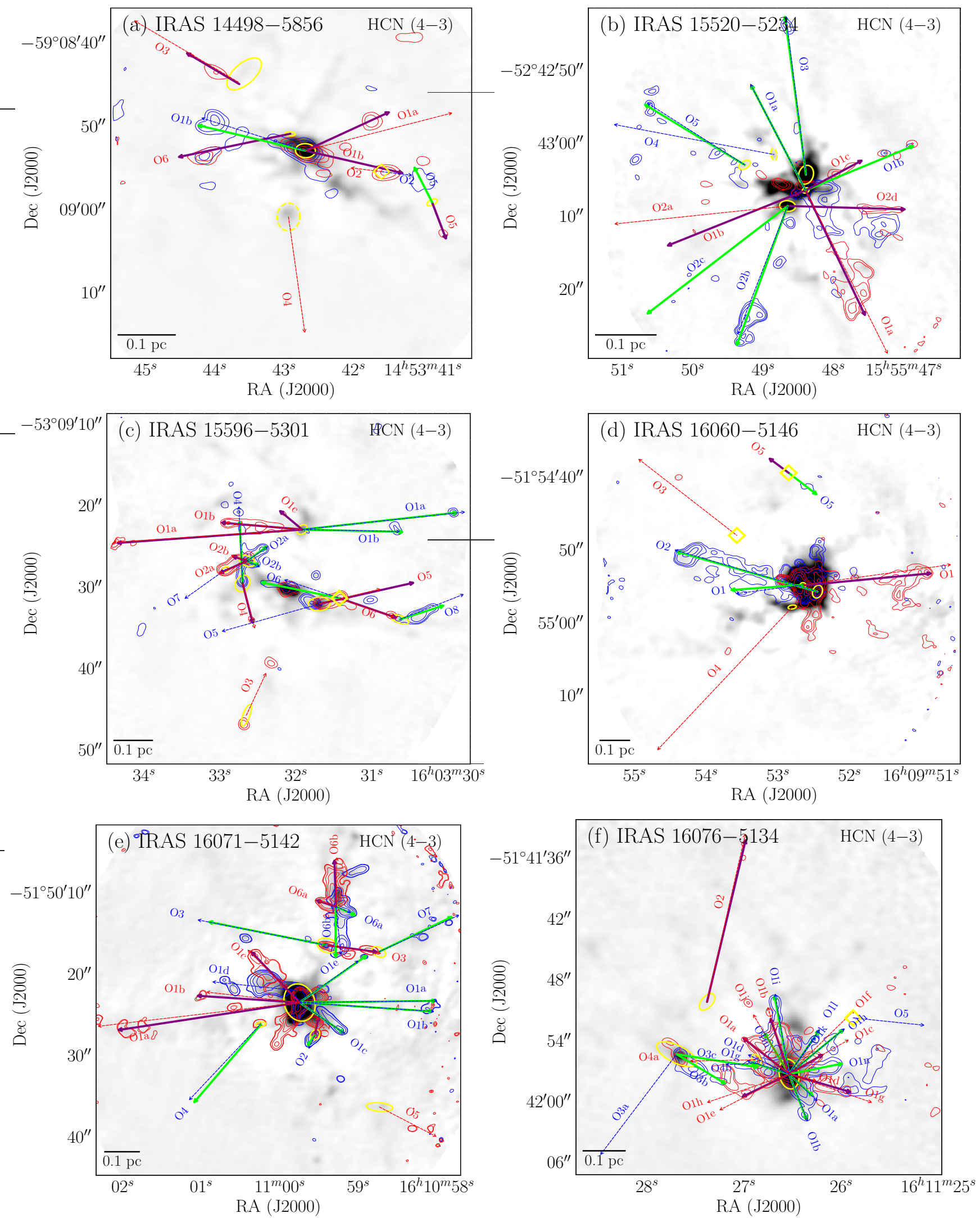

Figure A1. Images of HCN outflows in the six target fields. Background grey-scale images are the ALMA 0.9 mm continuum maps. The red and blue contours correspond to redshifted and blueshifted HCN gas integrated over carefully selected velocity ranges to depict the outflow lobes. The blueshifted and redshifted outflow lobes of HCN are marked by solid green and purple arrows, respectively, while the outflow lobes identified using CO are also shown by blue and red dashed arrows, respectively. The driving sources detected in the continuum map are marked in yellow ellipses. Outflow lobes with yellow diamonds are those for which no continuum sources are identified (see caption of Figure 1). 

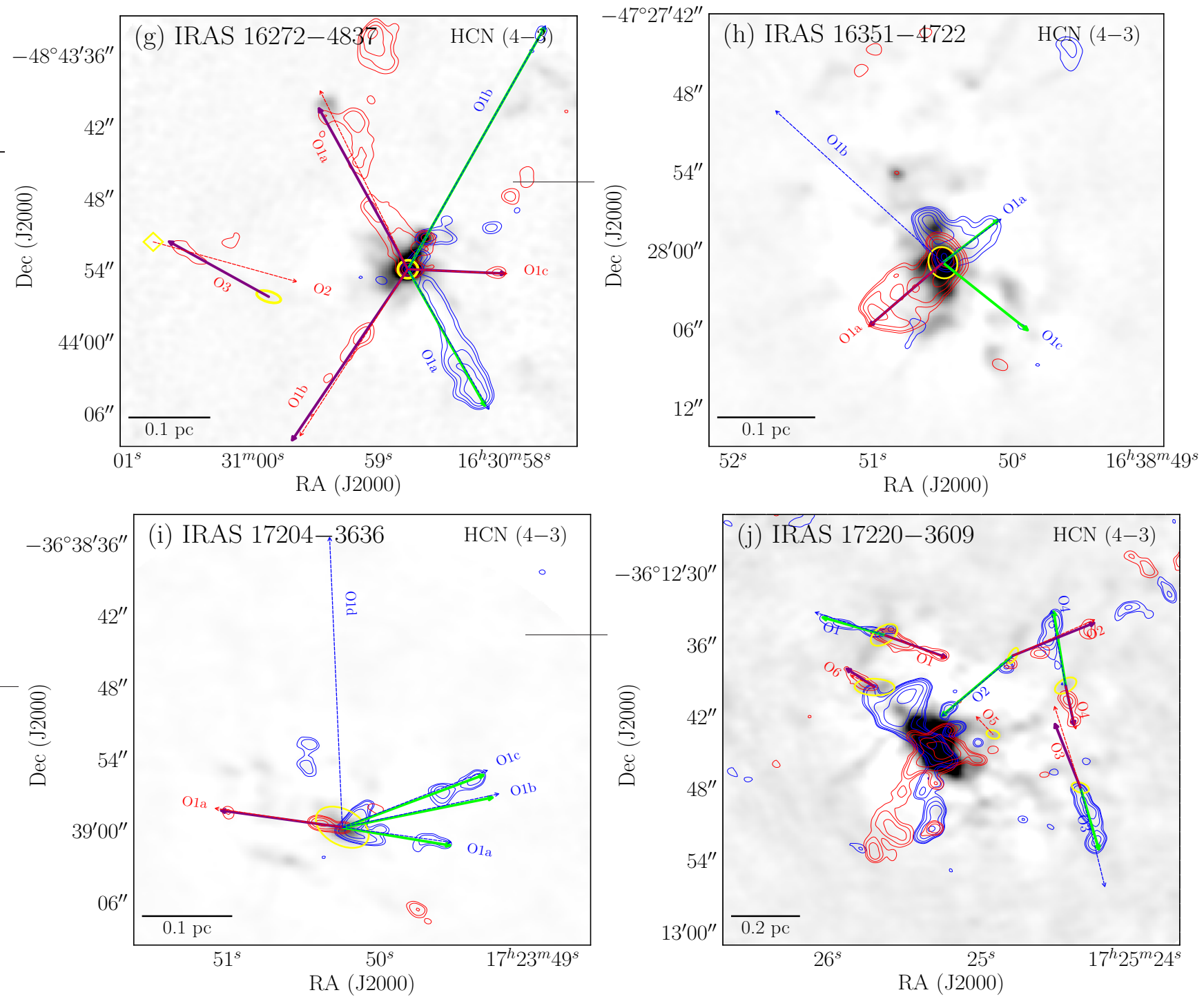

Figure A2. Images of HCN outflows for the remaining four target fields (see caption of Figure A1). 

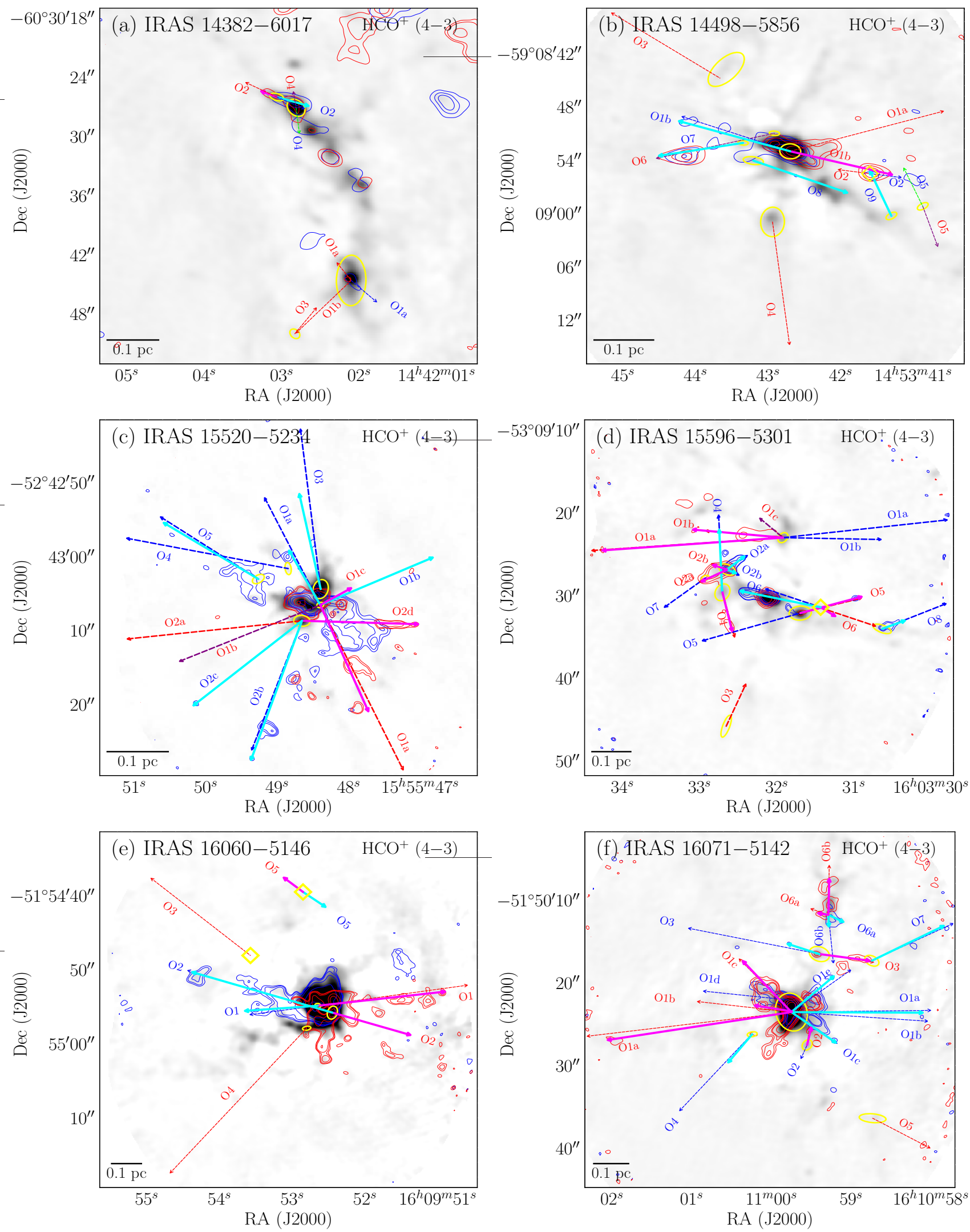

Figure A3. Images of $\mathrm{HCO}^{+}$outflows in six target fields. The red and blue contours correspond to redshifted and blueshifted $\mathrm{HCO}^{+}$gas integrated over carefully selected velocity ranges to depict the outflow lobes. The blueshifted and redshifted outflow lobes of $\mathrm{HCO}^{+}$are marked by solid cyan and magenta arrows, respectively. The blueshifted and redshifted outflow lobes identified using $\mathrm{CO}$ are shown by blue and red dashed arrows, while a few additional blueshifted and redshifted lobes that are identified only in HCN are marked by green and purple arrows, respectively. The remaining symbols are the same as shown in Figure 1 and Figure A1. 

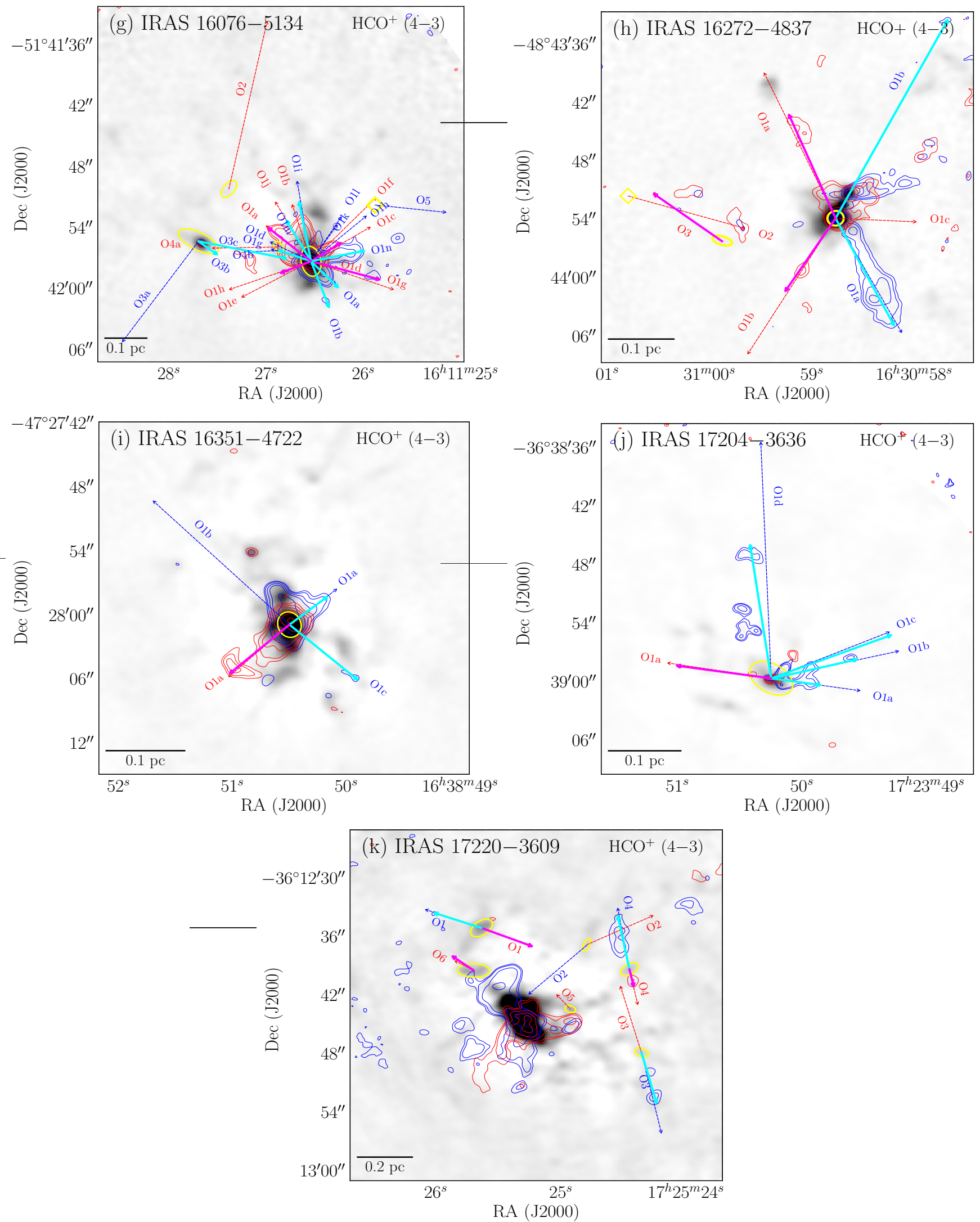

Figure A4. Images of $\mathrm{HCO}^{+}$outflows for the remaining five target fields. For details of the markers and symbols see caption of Figure A3. 